\title{
Measuring The Sources of Inflation in The Iraqi Economy Using a Model Self- Regression of Slowed Distributed Time-Laps (ARDL) Duration (1970-2018)
}

\author{
Ali Naba Sayel Al-Subaihi*,1, Nadhem Abid Al-Mihimdy ${ }^{1}$, Ahmed Hussein Battal Al-Ani ${ }^{2}$ \\ ${ }^{1}$ Department of Economics, College of Administration and Economics, University of Falluijah, Iraq \\ ${ }^{2}$ Department of Economics, College of Administration and Economics, University of Anbar, Iraq \\ * ali-nabaa@uofallujah.edu.iq
}

KEYWORDS: $\quad$ Inflation, ARDL, Statistical Analysis Software (Eviews9), Bound Test, Exchange Rate.

\begin{abstract}
:
Inflation posed a challenge to the Iraqi economy and its effects on economic activity and growth rates. This study investigates the sources of inflation in the Iraqi economy during the period (1970-2018) within the framework of the aggregate supply and aggregate demand model using the Self-Regression of Slow Distributed Gaps (ARDL) model. The results of the study showed that the inflation rate in Iraq is affected by the following variables: (money supply, parallel market exchange rate, consumer spending, the oil sector's contribution to GDP and the degree of economic openness) and all are related to an inverse relationship with the rate of inflation except for money supply and consumer spending, as It has a direct relationship with it, and there is a long-term equilibrium relationship (co-integration) between the studied variables according to the Bound Test methodology. Through the value of the error correction vector coefficient $(1.35 \%)$ of the short-term errors are automatically corrected during the unit time (year) to reach the equilibrium in the long term.
\end{abstract}

\section{REFERENCES:}

De vita G, Endresen K, and Hunt C., L (2005) "An Empirical Analysis of energy demand in Namibia" Department of Economics, University of Surrey.

Stock, J, and Watson, M., (2003) "Introduction to Econometrics" 2nd edition Addison Wesley Boston.

Dickey, D.A fuller (1981) "Likelihood Ratio Statistics for Autoregressive Time Series with a Unit Root", Econometrica, 1981, vol. 49, issue 4, 1057-72.

Narayna, P. k fijis Tourism demand (2004) "The ardel approach to cointegration tourism economics", vol (10), issue (2) Australia.

Diebold Francis (2016) Econometrics" first Edition University of Sylpenn Sylvania USA. 


\title{
قياس مصادر التضخم في الاقتصاد العراقي باستخدام أنموذج الإنحدار الذابي للفجوات الزمنية الموزعة المتباطئة (ARDL) للمدة (1970-2018)
}

\author{
أ.د. علي نبع صايل الصبيحي*،1، أ.د. ناظم عبدالله عبد المحمدي11، أ.د. أحد حسين بتال العالي2

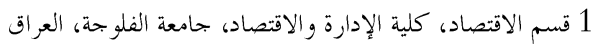

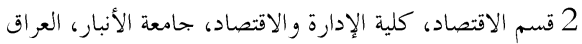 \\ *ali-nabaa@uofallujah.edu.iq
}
الكلمات المفتاحية ل التضخم، انموذج الانحدار الذايي للفجوات الزمنية الموزعة المثباطئة (ARDL)، برنامج التحليل الإحصائي (Eviews9)، اختبار الحدود، سعر الصرف.

\begin{tabular}{l|l} 
Crossref doi & https://doi.org/10.51345/.v32i2.343.g229
\end{tabular}

ملخص البحث:

شكل التضخم تحدياً للاقتصاد العر اقي وما له من آثار على النشاط الاقتصادي ومعدلات النمو ، وتقوم هذه الدراسة بالتحري عن مصادر التضخم في الاقتصاد العراقي خلال المدة (1970-2018) ضمن إطار انموذج العرض الكلي والطلب الكلي باستخدام انموذج الانحدار الذاتي للفجوات الموزعة المتباطئة (ARDL) وقد أظهرت نتائج الدراسة أن معدل التضخم في العراق يتأثر بالمتغيرات الآتية: (عرض النقد ، سعر الصرف في السوق الموازي ، الانفاق الاستهلاكي، مساهمة القطاع النفطي في الناتج المحلي الاجمالي ودرجة الانفتاح الاقتصادي) وكلها ترتبط بعلاقة عكسية مع معدل التضخم باستثناء عرض النقد ، والانفاق

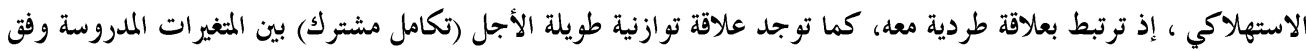
منهجية إختبار الحدود (Bound Test). ومن خحلال قيمة معامل متجه تصحيح الحطأ ان (1.35 \% من من أخطاء الأجل القصير يتم تصحيحها تلقائياً خلال وحدة الزمن (السنة) لبلوغ التوازن في الأجل الطويل.

المقدمة:

و اجه الاقتصاد العراقي مشكلات إقتصادية مختلفة ومنها مشكلة التضخم الاقتصادي وتمخض عنها نتائج ذات أبعاد إقتصادية واجتماعية خطيرة على مستوى القطاع الإنتاجي من جهة وعلى مستوى القطاع العائلي من جهة ثانية، فضلاً عن تأثيره في ابتحاهات سوق عناصر الانتاج المختلفة محا أثر سلباً في معدلات النمو الاقتصادي ويمكن التمييز بين مصلرين للتضخم هما العوامل الناشئة من جانب الطلب تغذيها السياسية النقدية والمالية التوسعية وعوامل جانب العرض النابحة عن ارتفاع تكاليف الإنتاج. فالمصدر الأول يندرج تحت ما يسمى بالمدرسة النقدية (Monetary School) في حين يندرج المصدر الثاني تحت مظلة المدرسة البنيوية (Structural School) فالمدرسة الأولى ترى أن التضخم ظاهرة نقدية بالدرجة الأولى، 
فالزيادة في عرض النقود تؤدي إلى زيادة نسبية في الأسعار المحلية، أما المدرسة البنيوية فإذا تركز على كلى القيود والمحددات التي تؤثر في جانب العرض كأحد أسباب النمو النقدي ومن ثم كمصدر للتضخهم، وتم قياس مصادر التضخم في الاقتصاد العراقي خلال مدة البحث من خلال إطار انموذج العرض الكلي

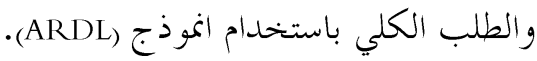

\section{مشكلة البحث:}

تتمثل مشكلة دراسة التضخم في الاقتصاد العراقي في تنوع وتشابك العوامل المسببة لتلك الظاهرة تبعاً لتنوع و تشابك الظروف التي احاطت بالاقتصاد العراقي وانعكاساها على سلوك المتغيرات الاقتصادية

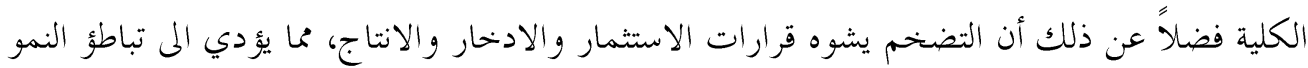
الاقتصادي وعرقلة تنوع الهيكل الاقتصادي.

\section{أهمية البحث:}

شكّل التضخم في الاقتصاد العراقي خلال العقود الماضية مشكلة للسياسة النقدية وقد اتخذ البنك

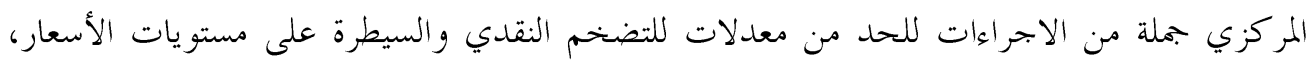
وعند قياس مصادر التضخم نحصل على تصور واضح للإجراءات التي تدعم السياسة النقدية.

\section{فوضيات البحث:}

1- يتأثر معدل التضخم في الاقتصاد العراقي سلباً وايجاباً بالتغيرات التي تحدث في عرض النقد، سعر الصرف في السوق الموازي، الانفاق الاستهلاكي، مساهمة القطاع النفطي في الناتج المحلي الاجمالي ودرجة الانفتاح الاقتصادي.

2- توجد علاقة توازنية قصيرة وطويلة الأجل بين معدل التضخم وعرض النقد، سعر الصرف في السوق الموازي، الانفاق الاستهلاكي، مساهمة النفط في الناتج، ودرجة الانفتاح الاقتصادي في الاقتصاد العراقي خلال مدة البحث.

\section{أهداف البحث:}

يهدف البحث إلى قياس مصادر التضخم في الاقتصاد العراقي للمدة (1970 - 2018) في إطار إنموذج العرض الكلي و الطلب الكلي باستخدام انموذج (ARDL). 


\section{منهجية البحث:}

بغية تحقيق أهداف البحث واختبار فرضياته فقد اعتمد البحث على المزج بين المنهج الوصفي والمنهج

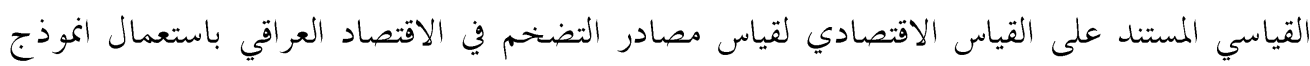
(ARDL) و باستخدام برنامج التحليل القياس (Eviews9)

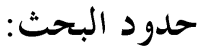

1. البعد الزماني: تناول البحث قياس التضخم للمدة (1970- 2018) ، علماً قسمت مدة البحث نهو الآتي: (1970- 1979، 1980- 1989، 1980،

لقد تعددت وجهات النظر حول أسباب التضخم منها ما استند إلى مفهوم النظرية الكمية ومنها ما

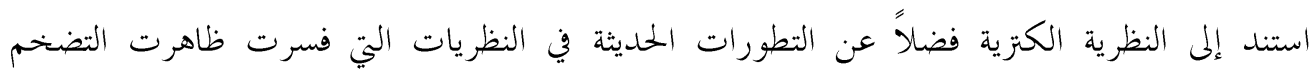
واستناداً إلى ذلك فقد تعددت التعريفات مع تعدد أسباب التضخهم ومصادره منها: وعرّف التضخم أيضاً بأنه: (حركة صعودية للأسعار تتصف بالاستمرار الذاتي تنتج عن فائض الطلب الزائد عن قدرة العرض) (داغر ، 2018: 199). و كذلك يعني حالة الارتفاع المستمر في الأسعار و يعكس حالة عدم التوازن ما بين القطاع النقدي والقطاع السلعي. وعرّف (Akly) آكلي التضخم: (بأنه عملية تعبر عن إبحاه واضح ومستمر في ارتفاع المستوى العام

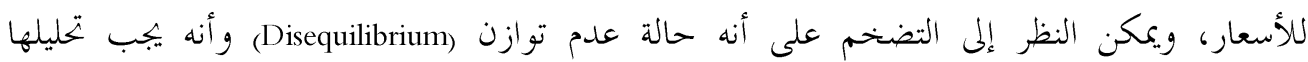

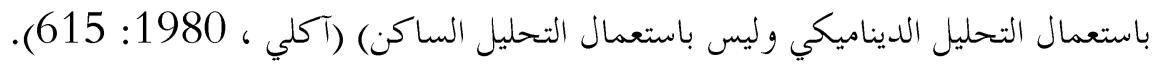

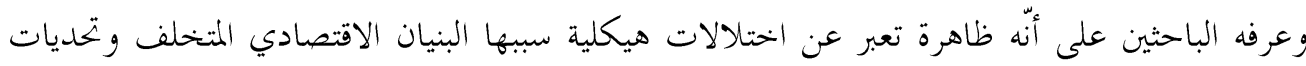

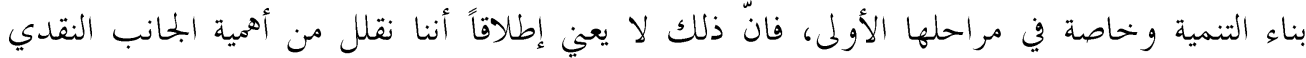

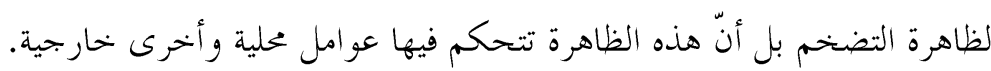

\section{مصادر التضخم في النظرية الاقتصادية}

يشكل التضخم أحد أهم مظاهر الاقتصاد العالمي المعاصر وقد حظيت دراسته باهتمام الباحثين وعلى الى الته

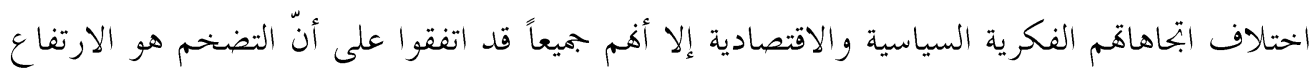


المستمر في المستوى العام للأسعار غير أنهم يعطون تعديلات وتشخيصات متباينة عند محاولتهم تفسير أسباب هذا الارتفاع وأصبح التضخم الهاجس الأساس لعدد غير قليل من دول العالم وخحاصة الدول النامية. إذ تفاقمت معدلات التضخم في السنوات الأخيرة وأخذت تؤثر على اقتصاداها وتضعف من معدلات النمو الاقتصادي فيها. أما مصادر التضخم فيمكن إجماها بالاتي: 1-التضخم الناشئ عن جذب الطلب Demand pull inflation. ترتفع الأسعار نتيجة لزيادة الطلب على العرض الكلي، ويكصل التضخم للوهلة الأولى بسبب زيادة عرض النقد، عندما يكون الاقتصاد في حالة الاستخدام الكامل ويؤدي إلى خفض سعر الفائدة محّا يؤدي

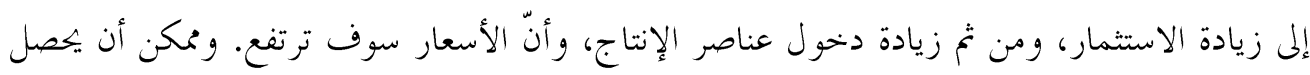
جذب الطلب من دون زيادة عرض النقد في حالة زيادة الكفاءة الحدية لرأس المال أو عندما يزداد الميل الحدي للاستهالك إذ يزداد الاستثمار (المعجل) مُّا يؤدي إلى زيادة الطلب الكلي عند ثبات العرض فان زيادة الطلب من D D إلى D Dودي إلى ارتفاع المستوى العام للأسعار من P P إلى P وان السياسات التي تخفض معدل الزيادة في الطلب الكلي او رفع معدل الزيادة في العرض الكلي او كليهما هي سياسات نقدية تخفض معدل نمو عرض النقد وسياسات مالية تقوم بها الحكومة من خلال خفض النفقات او زيادة الضرائب او الاثنين معاً، وهذه السياسات تخفض من حدة الضغط التضخمي على الاسعار، ان السياسة النقدية المقيدة او السياسة المالية او كليهما قد تؤدي الى زيادة البطالة خاصة اذا كان التضخم حدث لفترة طويلة من الزمن ولكن حالما يتهيء الاقتصاد للمعدل الجمديد من التضخم فان البطالة يجب ان تختفي (آكلي ، 1980: 378) 2- تضخم بلففع التكاليف Cost push inflation. يحدث التضخم بسبب ضغوط النقابات العمالية لزيادة الأجور النقدية بسرعة أكبر من الزيادة التي تحدث في الأحوال الاعتيادية، كما ينشأ عن الممارسات الاحتكارية للمديرين الذين يرفعون الأسعار في غياب الزيادة في الطلب أو ارتفاع النفقات (ابدجمان ، 1993: 379) وأنّ التضخم بدفع التكاليف الذي يحدث بسبب النقابات يسمى بالتضخم الناشئ عن ارتفاع الأجور Wage push inflation) بنسبة تفوق ارتفاع الإنتاجية مِّا يؤدي إلى ارتفاع الأسعار بينما التضخم الناشئ عن دفع التكاليف الذي ينشأ عن سلوك المنشآت يطلق عليه التضخم الناشئ عن زيادة الأرباح (Profit push inflation) إذ يعمل رجال الأعمال إلى زيادة نسبة الربح من خلال رفع الأسعار. وأنّ نقابات العمال عندما تطالب برفع الأجور 
فإنها تعتقد بأنّ ذلك يتم على حساب الأرباح، ولكن هذا الاعتقاد غير صحيح؛ لأنّ رجال الأعمال

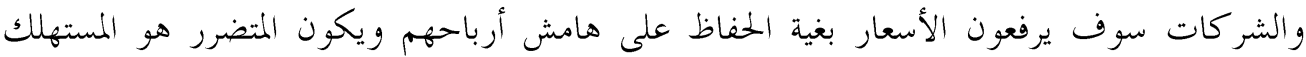

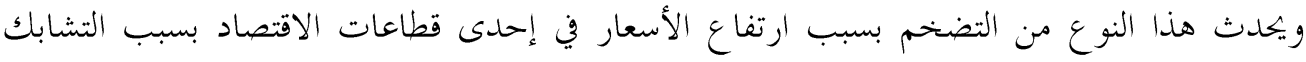

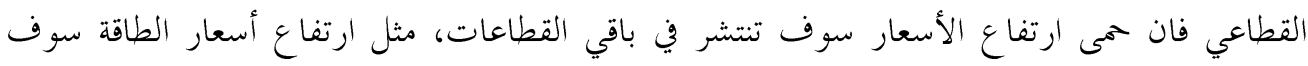

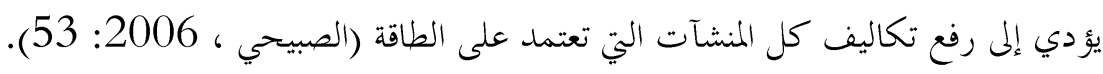
3- التضخم الهيكلي Structural Inflation.

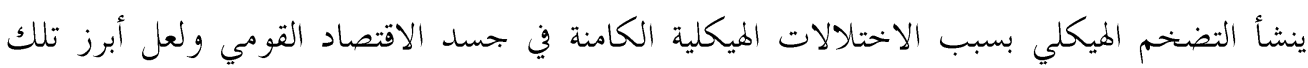

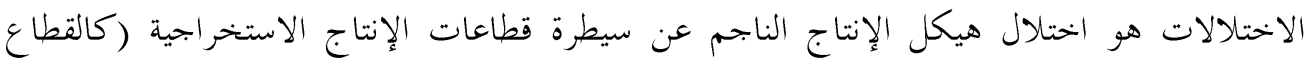

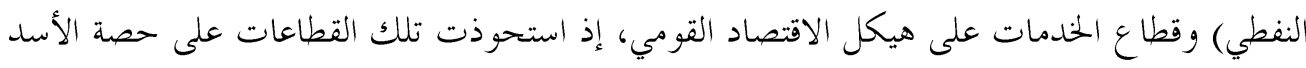

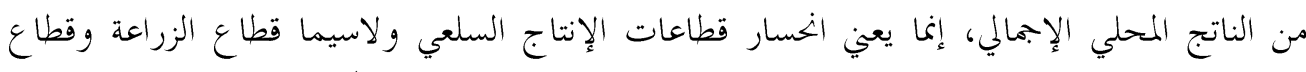

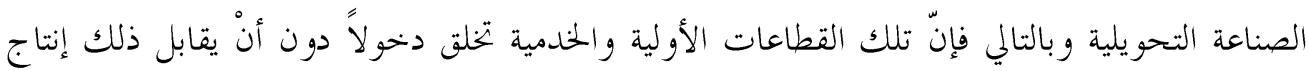

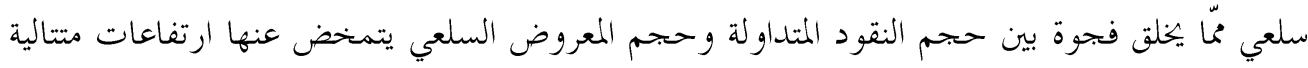

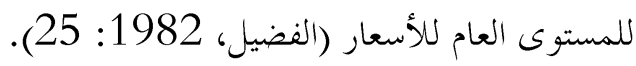

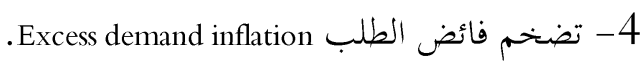

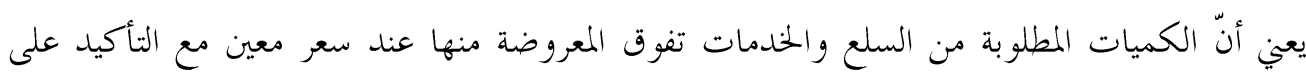

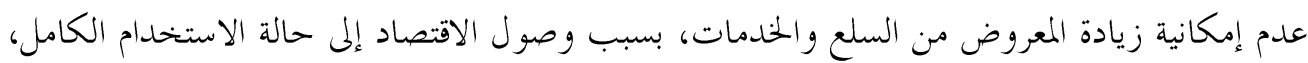

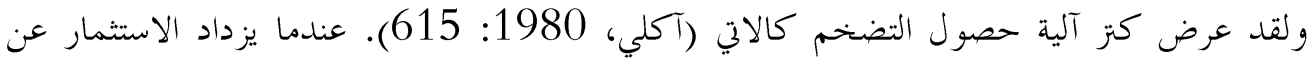

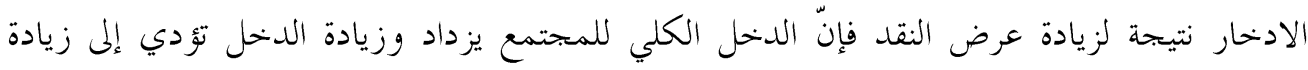

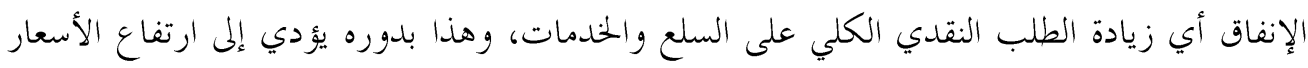

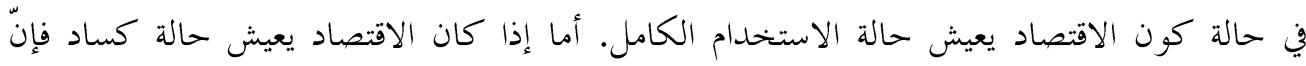

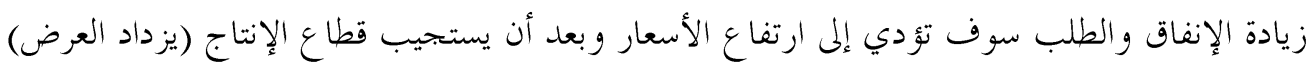

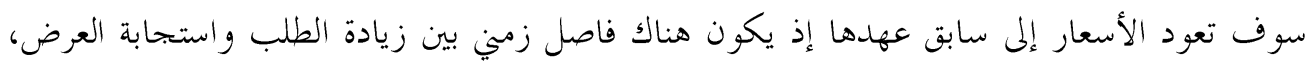

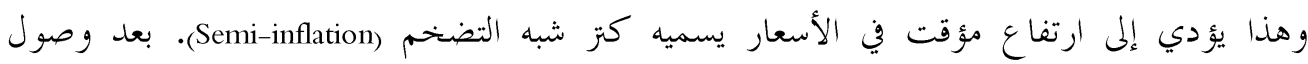

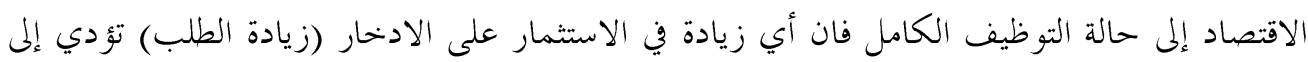

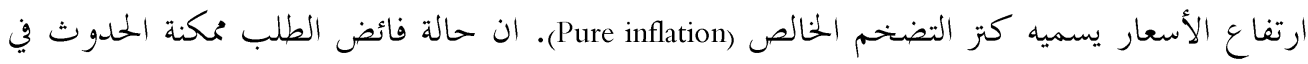

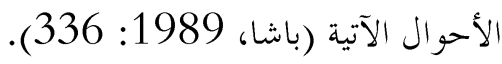


أ- في أوقات الحروب عندما يؤدي الإنفاق الحكومي إلى زيادة كبيرة في الطلب على مختلف السلع والخدمات. ب- في مدة التخطيط فان استثمارات القطاع العام تزداد و بالتالي فان الطلب الكلي يزداد.

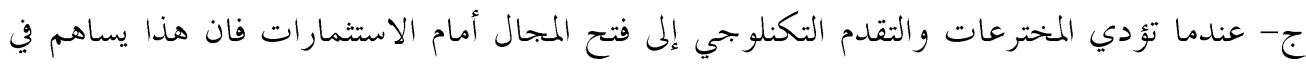
زيادة الطلب الكلي. د- يضاف إلى ذلك أنّ حدوث انخفاض في العرض الكلي غير مصحوب بانغفاض في الطلب الكلي يؤدي إلى حصول فائض في الطلب.

\section{الإطار النظري لبناء الانموذج القياسي والأساليب القياسية المستخدمة}

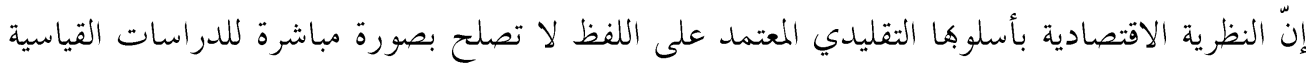

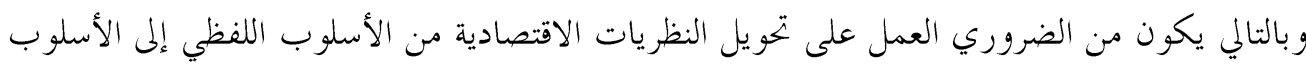

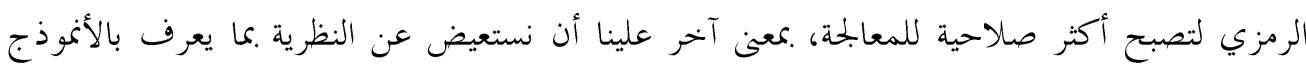

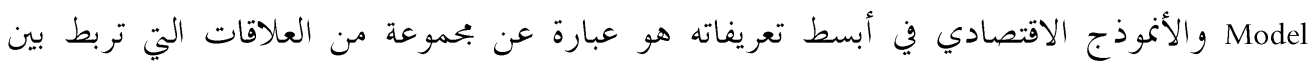

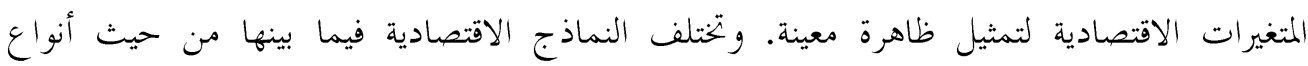
المتغيرات التي تشملها ومن حيث المعادلات التي تتضمنها. وقد تم قياس التضخم وفق الدالة نصف الوغاريتمية واعتماد ستة متغيرات مستقلة هي (M) تمثل كمية النقود، والإنفاق الكلي الاستهلاكي (XP) ومساهمة النفط في الناتج المحلي الإجمالي (OIL) ودرجة وستيل الانفتاح الاقتصادي (OP) وسعر الصرف (OX) وتم اضافة المتغير الوهمي (DD) للاستبعاد التقلبات التي حصلت خلال مدة الدراسة لطول الفترة الزمنية. وتعتبر النماذج القياسية اداة من ادوات التحليل الكمي التي تساعد في التعرف على حقيقة المتغيرات

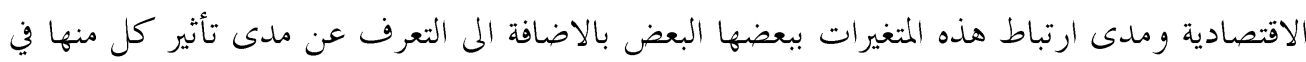

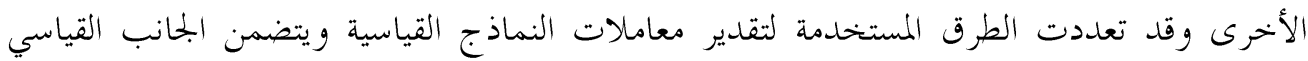
تقدير العلاقة بين التضخهم وعدداً من المتغيرات الاقتصادية المؤثرة فيه. ان الهدف من القياس هو التأكد من مدى صحة العلاقات بين متغيرات البحث مقارنة بما تستند اليه

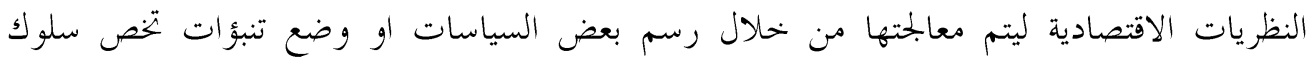

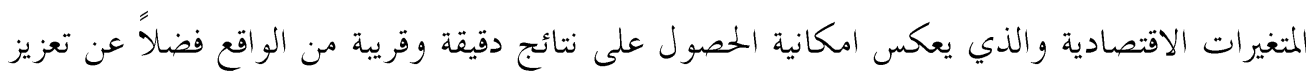

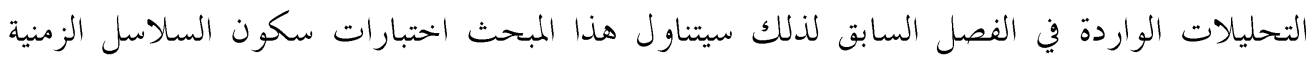


للمتغيرات الاقتصادية، واختبارات التكامل المشترك بين المتغيرات والذي يعد شرطاً لازماً لتطبيق

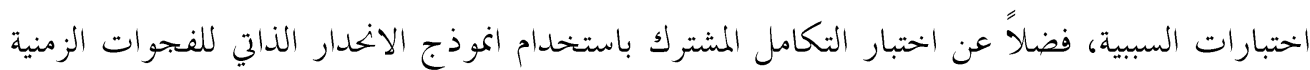
الموزعة المتباطئة (ARDL) لاختبار مدى وجود علاقة توازنية قصيرة وطويلة الأجل بين معدل التضخم ومصادره.

\section{Stationary of time series مفهوم سكون السلاسل الزمنية}

تعرف السلسلة الزمنية بأها بحموعة من البيانات المشاهدة المرتبطة فيما بينها والمسجلة خلال مدة زمنية

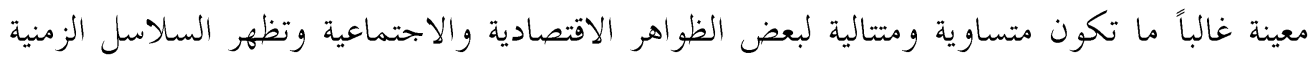
المتعلقة بالنشاط الاقتصادي احتو ائها على جذر الوحدة والذي يشير إلى أن متوسط وتباين المتغير غير

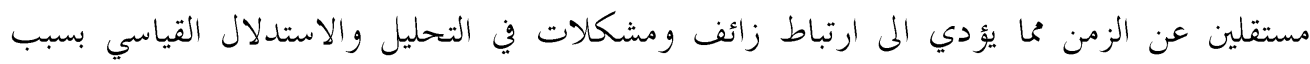

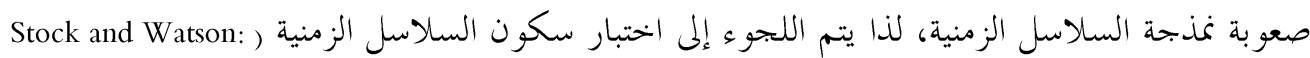

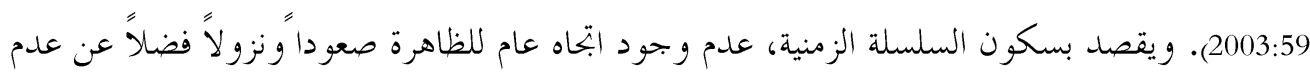

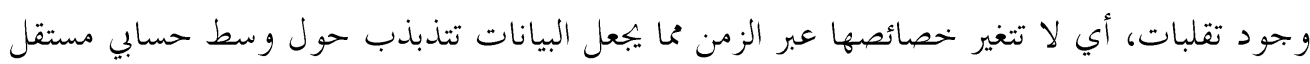
عن الزمن (الدليمي، مالك: 110:2018) ولابد من التحقق من سكون السلسلة الزمنية لكل متغير في

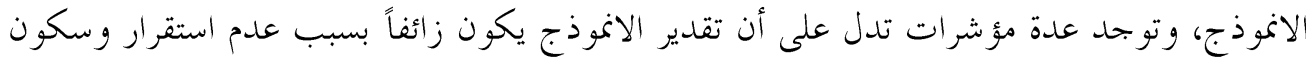
السلاسل الزمنية للمتغيرات الاقتصادية منها (العيسى: أ- وجود ارتباط تسلسلي ذاتي يبرز في قيمة دربن واتسن (D.W).

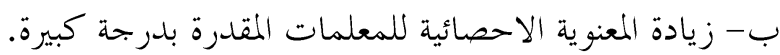

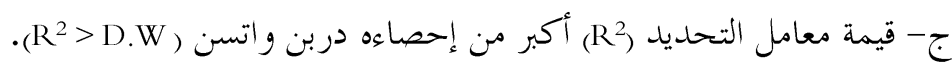

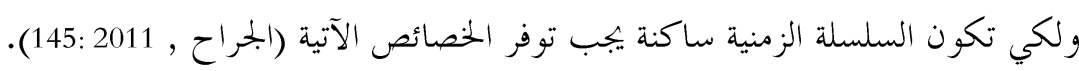

$$
\begin{aligned}
& \operatorname{Var}\left(Y_{t}\right)=E\left(Y_{t}-\mu\right)^{2}=\sigma^{2} \quad \ldots \text { (1) } \\
& \boldsymbol{E}\left(\boldsymbol{Y}_{\boldsymbol{t}}\right)=\boldsymbol{\mu} \ldots(2) \\
& \text { ب- ثبات متوسط قيم السلسة الزمنية }
\end{aligned}
$$

ج- قيمة التباين المشترك (Covariance) بين سلسلتين زمنيتين يعتمد على الازاحة (k) مما يعني ثبات التغاير و يعبر عن ذلك بالصيغة الاتية:

$\operatorname{COV}(\mathbf{Y t}, \mathbf{Y t}-\mathbf{k})=\mathbf{E}](\mathbf{Y t}-\boldsymbol{\mu})(\mathbf{Y t}-\mathbf{k}-\boldsymbol{\mu})[=\mathbf{\gamma K}$

اذ اذ ان - مان ب: الوسط الحسابي، (6) التباين , (8) معامل التباين المشترك (التغاير). 
إن سكون السلسلة الزمنية عند المستوى الأصلي (Level) أو عند الفرق الأول (15ifference) أو كليهما هو الذي يهاد نوع الانموذج أو اختبار التكامل المشترك المستخدم في القياس و التحليل. وبعد إجراء اختبارات السكون لبيانات السلاسل الزمنية يتحدد نوع الاختبار المناسب، وهناك عدة طرق لاختبار سكون السلاسل الزمنية منها: 1 1 التحليل البياني: يوضح الرسم البياني الزمني تصور أولى عن مدى سكون السلاسل الزمنية لأي متغير، فضلاً عن قدرته على بيان ابتحاه عام تصاعدي أو تنازلي للسلسلة الزمنية الناجم عن اختلاف متوسطات العينات الزمنية للسلسلة ككل، وهذا يعين عدم سكون السلسلة الزمنية ويصعب أحياناً تحديد طبيعة السلسلة، فيما إذا كانت ساكنة أو غير ساكنة بالرسم البياني، وعند ذلك تستخدم معايير أخرى منها دالة الارتباط الذاتي واختبارات جذر الوحدة (De Vita et al , 2006:34). 2- 2 اختبارات جذر الوحدة للسكون هدف إلى فحص خواص السلسلة الزمنية لكل متغير من متغيرات البحث خحلال المدة الزمنية للمشاهدات و التأكد من سكوفا وتحديد رتبة تكامل كل متغير على حدة، وإذا كانت السلسلة مستقرة في قيمتها الاصلية تكون متكاملة من الرتبة صفر (O)، وإذا استقرت السلسلة بعد أخذ الفرق الأول فإن السلسلة الأصلية تكون متكاملة من الرتبة الاولى (1)، وإذا استقرت بعد أخذ الفرق الثاني تكون السلسلة الأصلية متكاملة من الرتبة الثانية (رشاد , 268:2011).

ولأجل فهم اختبار ات جذر الوحدة لابد من التعرف على نوعين من السلاسل الزمنية غير الساكنة هما :(207: 2012 , شيخحي) أ- سلاسل زمنية غير ساكنة من النوع (Trend Stationary) (TC) توضح هذه السلاسل عدم سكونية تحديدية (Determinist) و تستخدم طريقة المربعات الصغرى (OLS) من أجل إعادةا للسكون و تأخذ الصيغة الآتية: $\mathbf{Y}_{\mathbf{t}}=\mathbf{F}\left(\mathbf{t}+\varepsilon_{\mathbf{t}}\right)$

ب):- (Difference Stationary) (DS) تظهر عدم سكونية عشوائية (Random) في مركبه الابحاه العام وتستخدم فيها معادلة الفرق الأول من أجل اعادةا للسكون و تأخذ الصيغة الآتية:

$$
Y_{t}=Y_{t-1}+\beta+\varepsilon_{t}
$$

وتنطلق نقطة البدء للكشف عن وجود جذر الوحدة في بيانات السلسلة الزمنية من خلال المعادلة الآتية: 


$$
\mathbf{Y}_{\mathbf{t}}=\rho \mathbf{Y}_{\mathrm{t}-\mathbf{1}}+\mu_{\mathrm{t}}
$$

$$
\text { اذ أن (-1 }
$$

حد الحطأ العشوائي يتوزع توزيعاً طبيعياً بوسط حسابي مساوي للصفر (E) =0 ثابت (Var(ut)=

\section{$\operatorname{Cov}\left(\boldsymbol{\mu}_{t}, \mu_{\mathbf{t}-\mathbf{k}}\right)=\mathbf{0}$}

وتوضح المعادلة (6) إجراء الانحدار لمتغير السلسلة الزمنية (yt) بو صفه متغير تابع مع المتغير نفسه، ولكن بتباطؤ مدة واحدة، فإذا كانت المعلمة (P) المقدرة تساوي واحد، فهذا يعين أن السلسلة الزمنية (y) تحتوي على جذر الوحدة وافتقادها لصفة السكون (عدم سكون السلسلة الزمنية) وهذه هي فكرة اختبار جذر الوحدة بصورة عامة (الدليمي، مالك: 113:2018). ومن أهم اختبارات جذر الوحدة

$$
\text { شيوعاً والتي سوف يتم توظيفها في البحث هي: }
$$

:Augmented Dickey - Fuller Test (ADF) اختبار ديكي فوللر الموسع هذا الاختبار أنشأ لتحليل طبيعة وخصائص السالاسل الزمنية عام (1981)، وهو أكفأ من اختبار جذر الوحدة البسيط لتصحيح مشكلة الارتباط الذاتي في البواقي عن طريق تضمين دالة الاختبار عدداً معين من فروقات المتغير التابع المتباطئ لتقدير واحدة او اكثر من معادلات الانحدار (الفراج، 2004: 66) الاتية:

$$
\begin{aligned}
\Delta Y_{t} & =B Y_{t-1}+\sum_{i=1}^{p} \lambda_{i} \Delta Y_{t-i}+u_{t} \\
\Delta Y_{t} & =a_{0}+B Y_{t-1}+\sum_{i=1}^{p} \lambda_{i} \Delta Y_{t-i}+u_{t} \\
\Delta Y_{t} & =a_{0}+a_{1} t+B Y_{t-1}+\sum_{i=1}^{p} \lambda_{i} \Delta Y_{t-i}+u_{t}
\end{aligned}
$$

اذ أن أن الفروق الأولى

( $\left.a_{0}, a_{1}, B\right)$ Y

P : عدد فترات الابطاء

Ut

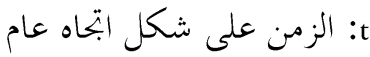

و.بمقارنة قيمة (t) المحتسبة مع قيمة (t) الجلدولية بعد تقدير الصيغ أعلاه باستخدام طريقة (OLS) فإذا كانت قيمة (t) المحتسبة أكبر من الجلدولية ترفض فرضية العدم (Ho: B=O) و تقبل الفرضية البديلة (H1:B) > O محا يدل على معنوية المعلمة الإحصائية وعلم وجود جأر الوحدة أي السلسلة الزمنية الساكنة و العكس صحيح، أما إذا كانت قيمة (t) المحتسبة أصغر من الجلدولية نقبل فرضية العدم (Ho: B=O) 
وترفض الفرضية البديلة (H1:B > O)، أي أن السلسلة الزمنية غير ساكنة، لذا نقوم بإجراء اختبار الفرق الاول للمعادلات السابقة، واذا ظهرت غير ساكنة نكرر الاختبار للفرق الثاني وهكذا ( Dickey \& Fuller $\cdot(250: 1981$

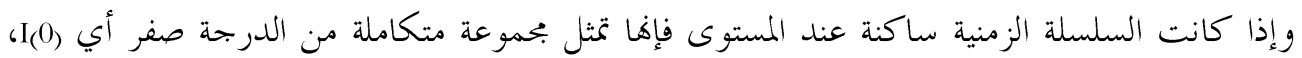
وإذا كانت غير ساكنة نأخذ الفرق الأول ويجري لها اختبار جذر الوحدة، وإذا تحقق لها صفة السكون

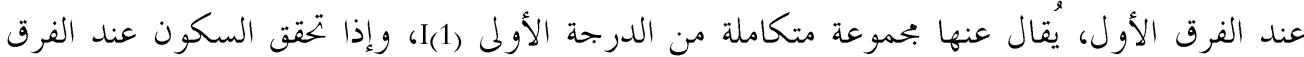

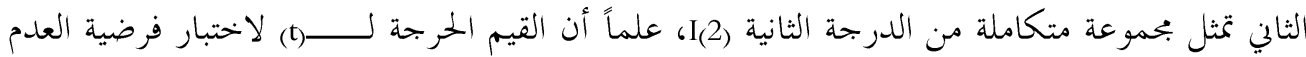
تعتمد على قيم ماكينون (Mackinnon). Phillips- Perron test (PP) اختبار فليبس بيرون يختبر فليبس بيرون الفرض الصفري وجذور الوحدة مقابل سكون الاتحاه أيضاً من خلال توظيف اختبار لا معلمي (Non Parametric) لصيغة ديكي فوللر غير المعدلة (التي لا يضاف إليها الفروق الاولى المتباطئة

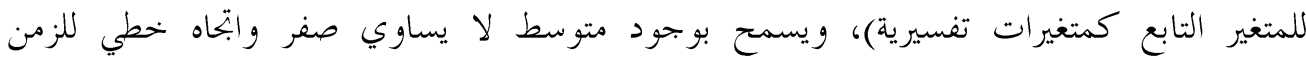
(79: 2017,سليمان) $\Delta \mathbf{y}_{t}=\mathbf{a}+\mathbf{B} \mathbf{y}_{\mathrm{t}-1}+\mathbf{u}_{\mathbf{t}}$ اذ أن: - د الفروق الأولى yt-1 القيم المتباطئة للمتغير محل الدراسة لمدة زمنية واحدة

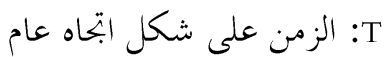
Ut ويختلف اختبار فليبس بيرون (pp) عن اختبار (ADF) كونه لا يحتوي على قيم متباطئة للفروق، ويعتمد

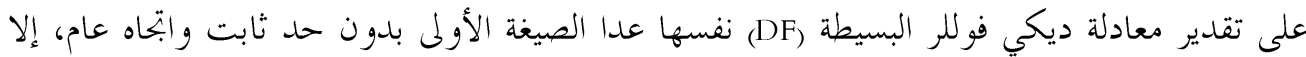
أنه يختلف عن اختبار (DF) في طريقة معالجة وجود الارتباط الذاتي من الدرجة الأولى:

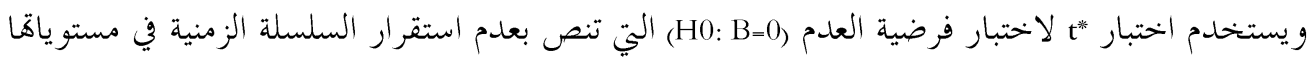
مقابل الفرضية البديلة التي تنص على سكون السلسلة الزمنية (H1: B> O) فعندما تكون قيمة (t) معنوية وهذا يعني رفض فرضية العدم وقبول الفرضية البديلة والتي تقضي بسكون السلسلة الزمنية (لا تحتوي

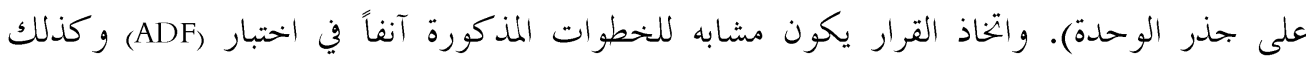

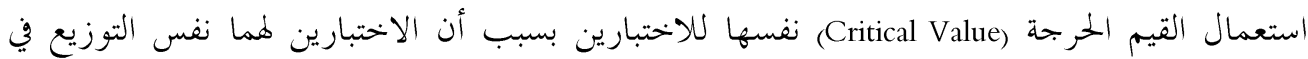
العينات الكبيرة (القدير: 211:2006). 
2- اختبار التكامل المشترك: Co-integration Test

تعتمد اختبارات التكامل المشترك على المفهوم الاقتصادي للخصائص الإحصائية للسالسل الزمنية ويتم الربط بين مفهوم التكامل المشترك ومفهوم النظرية الاقتصادية وخاصة فيما يتعلق بفكرة العلاقة التوازنية في الأجل الطويل، إذ ينص انموذج التكامل المشترك أن المتغيرات الاقتصادية التي تفترضها النظرية الاقتصادية تشير إلى وجود علاقة توازنيه بينها في الأجل الطويل، و لا تتباعد عن بعضها بشكل كبير في الأجل الطويل مع امكانية أن يختل هذا التوازن في الأجل القصير ويصحح هذا الاختلال في التوازن بقوى اقتصادية تقوم بإعادة هذه المتغيرات للتحرك نخو التوازن في الأجل الطويل (بتال وآخرون: 46:2014) و هناك عدة اختبار ات لتحليل التكامل المشترك. أ- اختبار أبخل - جر انبر ذي الخطوتين: تعتمد منهجية أبخل جر ابخر على خطو تين هما (Engel and Granger, 1988: 256) 1- يجب التحقق من سكون السلاسل الزمنية للمتغيرات الاقتصادية الملدروسة باستخدام أياً من اختبارات جذر الوحدة، إذا دلت النتائج على سكون السلاسل الزمنية للمتغيرات في مستوياتا الأصلية، أي أن المتغيرات متكاملة تكاملاً مشتر كا من الدرجة صفر (0)1 يمكن استخدام الطرق الإحصائية التقليدية للتقدير. وإذا كانت السلاسل الزمنية متكاملة من الدرجة الاولى (1)1 فإن: $\mathbf{Y}_{\mathbf{t}}=\mathbf{a}+\mathbf{B} \mathbf{X}_{\mathbf{t}}+\mathbf{e}_{\mathbf{t}}$ (11)

و تستخدم طريقة المربعات الصغرى OLS ثم يتم بعد ذلك اختبار استقرار البواقي (et) لمعرفة مدى سكون مقدرات سلسلة البواقي في العلاقة طويلة الأجل (et)، ومن نتائج المعادلة السابقة: إذا كانت العلاقة متكاملة تكاملاً مشتر كاً، ودلت نتائج تقدر المعادلة السابقة (11) على سكون البواقي فإن هذا يعني أن المتغيرين (y t, X t) متكاملان تكاملاً مشتركاً، أي وجود علاقة توازنية طويلة الأجل بينهما (سليمان، 2017 : 81). 2- حتسب البواقي والتي تقيس انحراف العلاقة المقدرة في الأجل القصير عن ابتحها التوازني في الأجحل الطويل وفق المعادلة الآتية (عطية ,688:2005). 3- بعد الخصول على بواقي الانحدار المقدر (Ût) الناجمة عن انحدار العلاقة التوازنية طويلة الأجل يتم اجراء احدى اختبارات السكون على بواقي الانحدار و كالاتي: $\triangle \hat{\mathbf{U}} \mathbf{t}=\mathbf{b} 0+\mathbf{b} 1 \hat{\mathbf{U}} \mathbf{t}-\mathbf{1}+\Delta \hat{\mathbf{U}} \mathbf{t}-\mathbf{1}+\mathbf{e t}$ 
بعد تحديد قيمة (t) المحتسبة نقارها بالقيمة الحرجة لها من جداول اعدها انجل وجرانجر واذا تم قبول فرضية العدم (HO: B=0) فهذا يعني وجود جذر الوحدة في سلسلة البو اقي اي اهذا غير ساكنة مما يدل على عدم وجود تكامل مشترك بين السلاسل الزمنية للمتغيرات المكونة للانموذج واذا تم قبول الفرضية البديلة (H1: B1 =0) للانموذج تتصف بخاصية التكامل المشترك اي وجود علاقة توازنية طويلة الأجل بينهما مما يجعل انموذج تصحيح الخطأ (ECM) (Error Correction Model) (Eثر ملائمة لتقدير العلاقة بينهما (الزهراني،

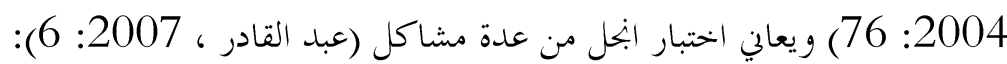

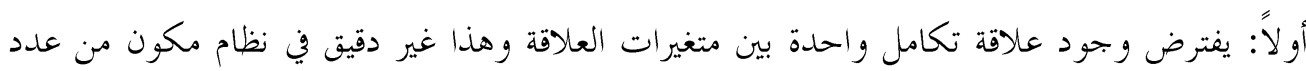
كبير من المعادلات. ثانياً: يحدد التكامل المشترك بابتاه واحد لمتغيرين فقط وهذا يمثل قيداً كبيراً عند وجود اكثر من متغيرين مع العلاقات التبادلية. نالثاً: يفترض ان احد المتغيرات متغير تابع وباقي المتغيرات تعد مستقلة.

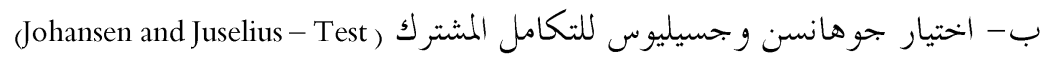

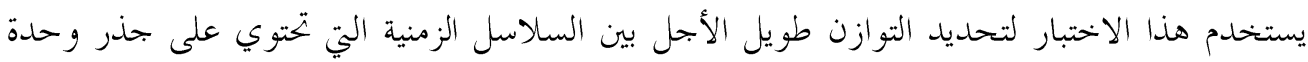

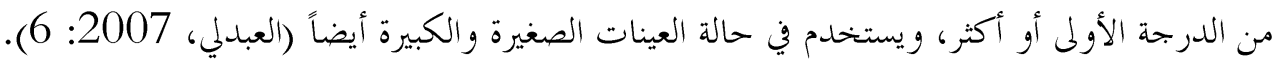
أن اختبار جوهانسن يتفوق على اختبار أبخل وجرانجر ويتناسب هذا الاختبار مع العينات الصغيرة

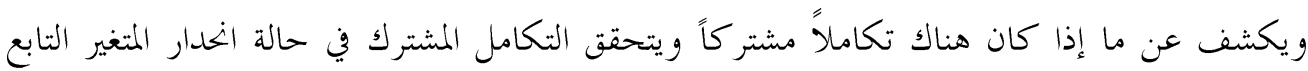

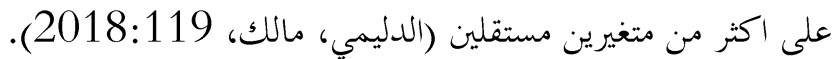

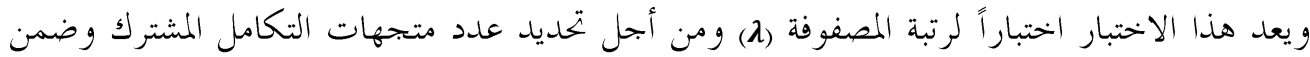
سياق اختبار جوهانسن يتم استخدام اختبارين احصائيين مبنيين على دالة الامكانيات العظمى (LR) (Like Lihood Ratio Test 1- اختبار الأثر (Trace Test) ويأخذ الصيغة الاتية:-

$$
\lambda_{\text {trace }}=-T \sum_{i=r+1}^{n} \log \left(\hat{\lambda}_{i}\right)
$$

اذ اذ ان - مان

r عدد متجهات التكامل المشترك القيم الذاتية لمصفوفة التباين والتباين المشترك ، T: حجم العينة. 


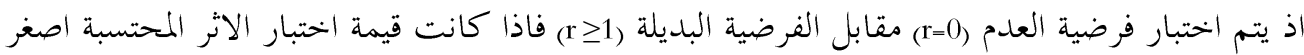
من القيمة الحرجة تقبل فرضية العدم واليت تعني ان متجهات التكامل المشتر تساوي صفر، اما اذا كانت قيمة الاختبار المحتسبة اكبر من القيمة الحرجة، تقبل الفرضية البديلة والتي تعبي ان عدد المتجهات اكبر من الصفر مما يعين وجود تكامل مشترك بين متغيرات النموذج (الجنابي ، 2012: 64). 2- اختبار الامكان الأعظم Maximum Eigenvalues Test (Max) (يوسفات، 2006:9) ويكسب اختبار الامكان الأعظم بالصيغة الآتية:

$\lambda_{\text {Max }}=-T \log \left(1-\hat{\lambda}_{i}\right)$

ند اختبار فرضية العلدم مقابل الفرضية البديلة يتم مكقارنة قيمة (t) مع القيمة الخرجة عند مستوى معنوية معينة استناداً إلى جدول جوهانسن وجيسليوس ، فاذا كانت قيمة (t) لمعدل الامكان الاعظم اكبر من القيمة الحرجة ترفض فرضية العلدم وتقبل الفرضية البديلة مما يعين وجود تكامل مشترك بين المتغيرات و معرفة عدد متجهات هذا التكامل و العكس صحيح في حالة قبول فرضية العلدم ورفض الفرضية البديلة محا يعين عدم وجود تكامل مشترك (العيثاوي ، 2020: 98). 3- اختبار التكامل المشترك باستخدام انموذج الانحدار الذاتي للإبطاء الموزع:

\section{Autoregressive Distributed Lag Model (ARDL)}

تتطلب اختبارات التكامل المشترك (أبخل، وجرابخر، جوهانسن، وجسيليوس) أن تكون المتغيرات متكاملة من الرتبة نفسها وتنتج عنها نتائج غير دقيقة إذا كان عدد المشاهدات صغيراً وبسبب هاتين المشكلتين أصبح منهج الانحدار الذاتي للابطاء الموزع (ARDL) شائع الاستخدام في المدة الأخيرة والذي قدم من قبل بيسران و آخرون (Pesaran et. al) عام (2001) وفي هذا النموذج تكون السلسلة الزمنية دالة في ابطاء قيمها وقيم المتغيرات المستقلة الحالية وابطائها بمرة واحدة او اكثر وتتميز منهجية ARDL) بعدة

$$
\text { مزايا أهمها (الشوربيجي، 2009: 1569): }
$$

1 - لا يستلزم اختبار (ARDL) ان تكون السلسلة الزمنية متكاملة من الرتبة نفسها (0)1 او من الرتبة (1)1 بشرط أن لا تكون متكاملة من الرتبة الثانية (2) فقط. 2- امكانية تقدير الأجل الطويل والقصير في آن واحد فضلاً عن امكانية التعامل مع المتغيرات التفسيرية في النموذج بفترات ابطاء زمنية مختلفة. 3- أنموذج (ARDL) يعطي أفضل النتائج للمعلمات في الأجل الطويل ، واختبارات التشخيص يمكن (Narayan \& fijis , 2004: 205) (الاعتماد عليها 
4- من خحلال انموذج (ARDL) ممكن تحديد العلاقة التكاملية بين المتغير التابع والمتغيرات المستقلة فضلاً عن عدم تحديد حجم تأثير كلاً من المتغيرات المستقلة على المتغير التابع وتعد معلماته المقدرة للأحلين

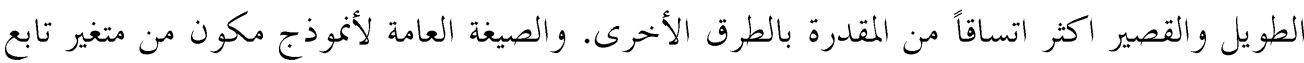
$\operatorname{ARDL}\left(\mathrm{P}, \mathrm{q}_{1}, \mathrm{q}_{2}\right.$

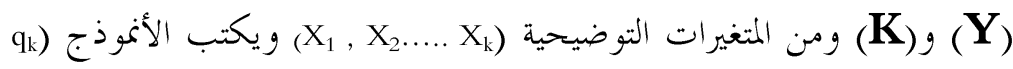
بالشكل الآتي: (حسن، :188:2013) ونعن (188).

$$
\begin{aligned}
& \Delta \mathbf{Y}_{\mathrm{t}}=\mathbf{c}+\mathbf{B}_{1} \mathbf{Y}_{\mathrm{t}-1}+\mathbf{B}_{2} \mathbf{X}_{1 \mathrm{t}-1}+\mathbf{B}_{3} \mathbf{X}_{2 \mathrm{t}-1}+\ldots \ldots+\mathrm{B}_{\mathrm{k}+1} \mathbf{X}_{\mathrm{kt}-1}+\sum_{\mathrm{i}=1}^{\mathrm{p}-1} \lambda_{1 \mathrm{i}} \Delta \mathbf{Y}_{\mathrm{t}-\mathrm{i}}+\sum_{\mathrm{i}=\mathbf{0}}^{\mathrm{q}_{1}-1} \lambda_{2 \mathrm{i}} \Delta \mathbf{X}_{1 \mathrm{t}-\mathrm{i}} \\
& +\sum_{i=0}^{q_{2}-1} \lambda_{3 i} \Delta \mathbf{X}_{2 t-i}+\ldots \ldots+\sum_{i=0}^{q_{k}-1} \lambda_{(k+1) i} \Delta \mathbf{X}_{k t-i} \\
& +\mu_{\mathrm{t}} \ldots \ldots \ldots \ldots \ldots \ldots
\end{aligned}
$$

إذ إن:

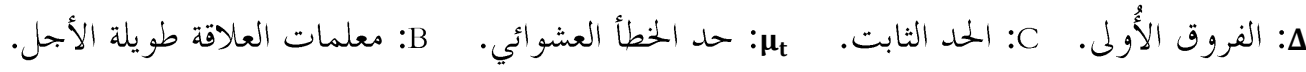
ג: معلمات العلاقة قصيرة الأجل.

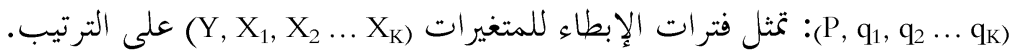
وبعد تحديد درجة تكامل متغيرات الانموذج وفق اختبارات (ADF, PP) وتحديد فترات الابطاء المثلى ومن أجل تطبيق اختبار التكامل المشترك في اطار منهجية انموذج الانحدار الذاتي للفجوات الزمنية المتباطئة

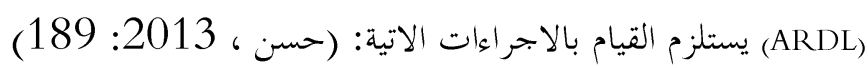

\section{اولاً: منهج إختبار الحمدود Bounds Testing Approach}

يستخدم لإختبار مدى وجود علاقة توازنية طويلة الأجل بين المتغير التابع والمتغيرات التفسيرية الداخلة

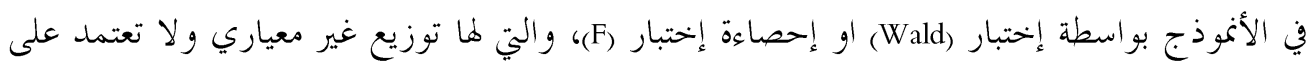

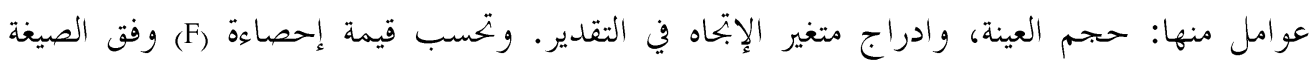
(Diebold, 2016: 43) - الآتية

$\mathbf{F}=\frac{(\mathbf{S S e R}-\mathbf{S S e u}) / \mathbf{M}}{\mathbf{S S e u} /(\mathbf{n}-\mathbf{k})}$

إذ إن:

:SSeR طويلة الأجل بين المتغيرات (إنعدام التكامل المشترك بين المتغيرات).

$\mathrm{H}_{0}: \mathrm{B}_{1}=\mathrm{B}_{2}=$ $=\mathrm{B}_{\mathrm{K}+1}=\mathbf{0}$ 
SSeu طويلة الأجل بين المتغيرات (وجود تكامل مشترك بين المتغيرات). $\mathrm{H}_{1}: \mathrm{B}_{1} \neq \mathrm{B}_{2} \neq \ldots \ldots \ldots \ldots \ldots \mathrm{B}_{\mathrm{K}+1} \neq \mathbf{0}$

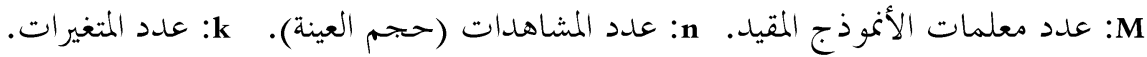
وبعد إحتساب قيمة إحصاءة (F) تتم مقارنتها بقيمة (F) الجلدولية المحتسبة من قبل (Pesaran et.al) عام

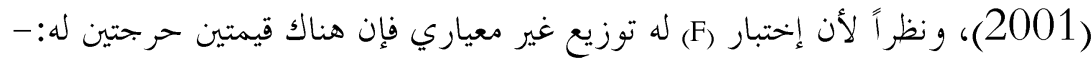
- ميمة الحد الأدلن:- و تفترض أن كل المتغيرات ساكنة في مستواها الأصلي، أي متكاملة من الرتبة صفر (0)

- - قيمة الحد الأعلى:- وتغترض أن كل المتغيرات ساكنة في فرقها الأول، أي متكاملة من الرتبة

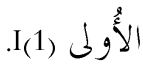

$$
\begin{aligned}
& \text { ويكون القرار على ثلاث حالات:- (حسن، 2013: 190) }
\end{aligned}
$$

1 إذا كانت قيمة إحصاءة (F) المحتسبة أقل من قيمة الحد الأدلى لقيمة (F) الجدولية، تقبل فرضية

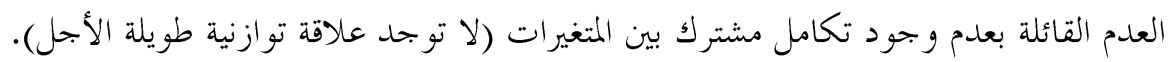

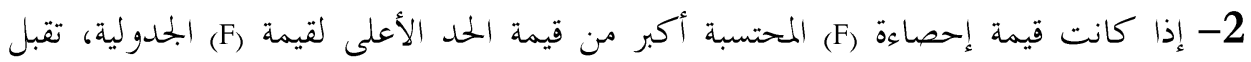
الفرضية البديلة القائلة بوجود تكامل مشترك بين المتغيرات (وجود علاقة توازنية طويلة

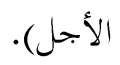

3- إذا كانت قيمة إحصاءة F) المحتسبة تقع بين الحدين الأعلى والأدلى لقيمة (F) الجلدولية، فان

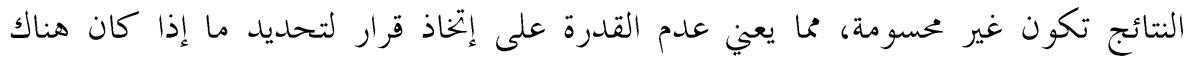

$$
\text { تكامل مشترك بين المتغيرات من علدمه. }
$$

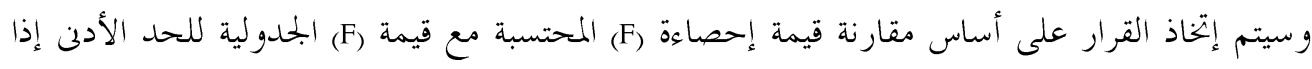

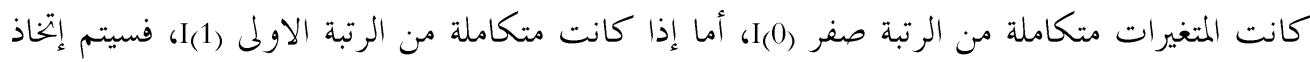
القرار على أساس مقارنة قيمة إحصاءة (F) المحتسبة مع قيمة (F) الجحدولية للحد الأعلى.

ثانياً: تقدير معلمات أنموذج (ARDL) للأجل القصير والطويل ومعلمة تصــحيح الحططــأ (VECM)

بعد التأكد من وجود علاقة توازنية طويلة الأجل بين المتغير التابع والمتغيرات التفسيرية، يتم تقدير

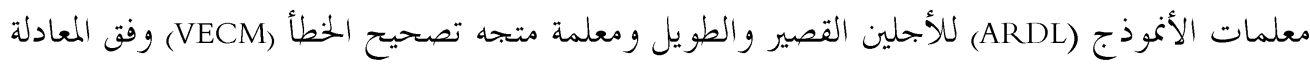


(16) بإستخدام طريقة المربعات الصغرى الإعتيادية (OLS) إستناداً إلى علد فترات الإبطلاء المحددة، ولتحديد الأنموذج الملائم يتم الإعتماد على طريقة (Hendry) الذي ينتقل من العام إلى الخاص، والذي يتمثل في إلغاء متغير الفروق الأُولى لأي متغير تكون القيم المطلقة لإحصاءة (t) الخاصة به غير معنوية أي أقل من الواحد الصحيح (حسن، 2013: 189). وقبل إعتماد هذا الأنموذج المقدر وتطبيقه ينبغي التأكد من جودة أداء هذا الأنموذج، ويتم ذلك من خلال إجراء الإختبارات التشخيصية الآتية:(الشوربجي، 159: (1509)

1- إختبار مضروب لاكر انج لإربتباط التسلسلي بين البواقي (BGLM).

\section{Lagrange Multiplier Test of Residual \{Brush-Godfrey\}}

$$
\text { 2- إختبار عدم ثبات التباين المشرووط بالإنحار الذاتي. }
$$

Autoregressive Conditional Heteroscedasticity $(\mathrm{ARCH})$

$$
\text { 3arque Bera JB) إختبار التوزيع الطبيعي للأخطاء العشوائية. }
$$

\section{ثالثاً : إختبار إستقرارية معلمات أنموذج (ARDL):}

لكي يتم التأكد من خلو البيانات المستخدمة في البحث من وجود أي تغيرات هيكلية فيها، ومعرفة مدى إستقرار وإنسجام معاملات الأجل الطويل مع تقديرات معلمات الأجل القصير يمكن إستخدام أحد

$$
\begin{aligned}
& \text { الإختباريين الآتيين:-- (ادريوش وعبد القادر، 2013: 23). } \\
& \text { 1- إختبار المجموع التراكمي للبو اقي المعاودة. }
\end{aligned}
$$

\section{Cumulative Sum of Recursive Residual (CUSUM)}

$$
\text { 2- إختبار المجموع التراكمي لمربعات البواقي المعاودة. }
$$

\section{Cumulative Sum of Squares Recursive Residual (CUSUM-SQ)}

و اُعد الإختبارين من أهم الإختبارات في هذا المجال، لأهما يوضحان أمرين مهمين هما: مدى إستقرار و إنسجام المعلمات طويلة الأجل مع معلمات الأجل القصير، و توضيح مدى وجود أي تغير هيكلي في البيانات. ووفقاً لهذين الإختبارين يتحقق الإستقرار الهيكلي للمعلمات المقدرة بصيغة تصحيح الخطأ للأنموذج (ARDL) إذا وقع الخط البياني لإختبار كل من (CUSUM) و (CUSUM-SQ) داخل الحلدود الحرجة (الحد الأعلى والحد الأدن) عند مستوى معنوية (5\%)، في حين تكون المعامالات لا تتسم بالإستقرارية الهيكلية إذا وقع الخط البياني للإختبارات خارج الحلدود الحرجة عند مستوى معنوية

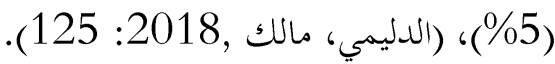




\section{نتائج قياس وتحليل مصادر النضخم في الاقتصاد العر اقي للمدة (1970- 2018):}

\section{توصيف الأنموذج القياسي:}

يمكن بناء انموذج قياسي لقياس مصادر التضخم في الاقتصاد العراقي طبقاً للتصورات الاتية:-

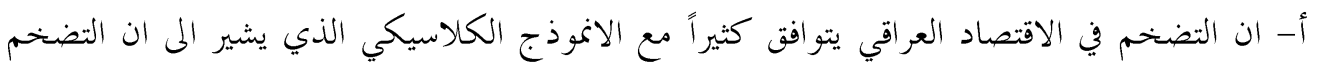
دالة لكمية عرض النقود.

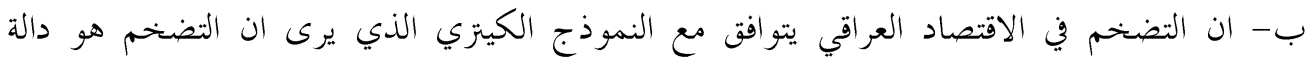

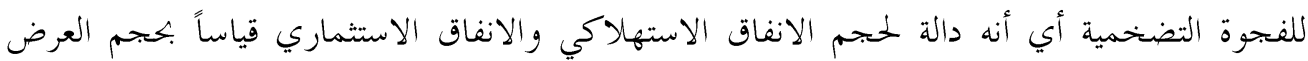

$$
\text { الكلي (الناتج المحلي الاجمالي). }
$$

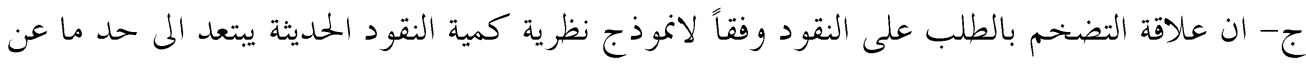

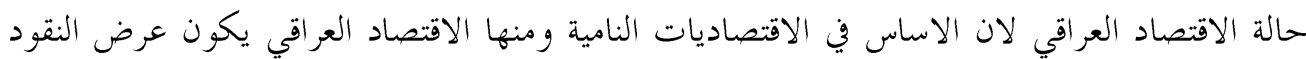

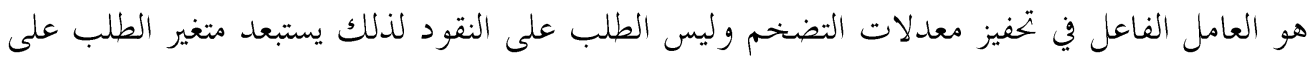
النقود من الانموذج القياسي. د- ان انموذج التضخم طبقاً للمدرسة السويدية يتو افق كثيراً مع حالة الاقتصاد العراقي اذ ان التضخم التحم

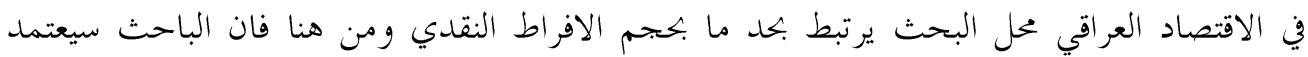
نصيب الوحدة الواحدة من الناتج المحلي الاجمالي من كمية النقود المتداولة كاحد المتغيرات المحددة لمدية المعدل التضخم في الاقتصاد العراقي. هـ- وبصدد قياس أثر الاختلالات الهيكلية في التضخم طبقاً للألموذج الهيكلي فان الباحث يختلف قليلاً عن رؤية ذلك الانموذج في تشخيص مسببات ارتفاع الاسعار في العراق اذ ان مصدر الاختلالات الهيكلية في الاقتصاد العراقي ناجم عن اعتماده شبه الكامل على القطاع النفطي ومن هنا اعتمد الباحث على مساهمة القطاع النفطي في الناتج المحلي الاجمالي كونه مصدراً من مصادر التضخم في الاقتصاد العراقي. ويُقصد بتوصيف النموذج صياغة العلاقات الإقتصادية بين المتغيرات موضوع الدراسة في صورة رياضية

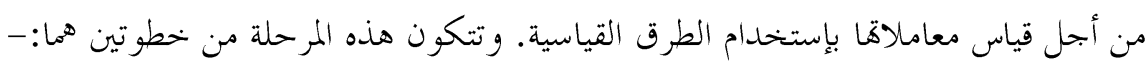




\section{تحديد متغيرات الأنموذج والإشارات المسبقة للمعالم:}

بناء" على التحليل الإقتصادي لمصادر التضخم والمعلومات المتاحة عن الدراسات القياسية السابقة، يمكن صياغة الأنموذج القياسي لمصادر التضخم والعوامل المؤثرة فيه وتحديد التوقعات النظرية المسبقة عن اشارات معالم النموذج وفق العلاقة الدالية الآتية:-

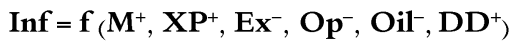

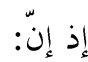

inf M : عرض النقد، ويفترض انه يرتبط بعلاقة طردية مع التضخم.

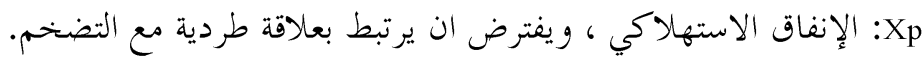
Ex

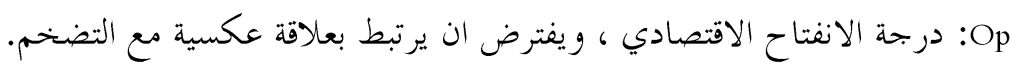

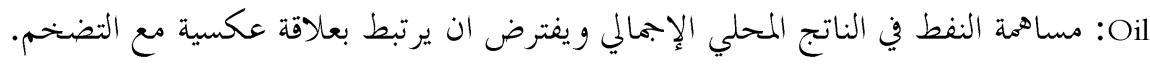
المتغير الو همي لاستبعاد أثر التقلبات التي حصلت خلال مدة البحث بسبب طول المدة الزمنية.

\section{إختيار الصيغة الرياضية المناسبة:}

يعتبر الأُسلوب الكمي وسيلة مهمة في تفسير أبعاد النظرية الإقتصادية على مستوى الإقتصاد الكلي

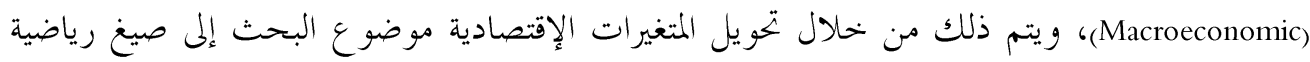

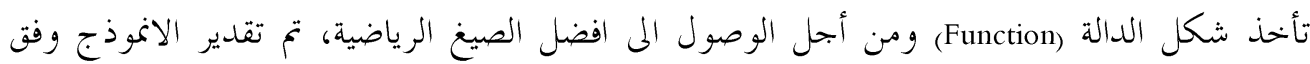
طريقة المربعات الصغرى الاعتيادية (OLS) باستخدام الصيغة الخطية والنصف اللوغارتمية واستبعاد الصيغة اللوغارتمية المزدوجة كون بعض قيم المتغير المعتمد (معدل التضخم) تحتوي على قيم سالبة. وتم اختيار

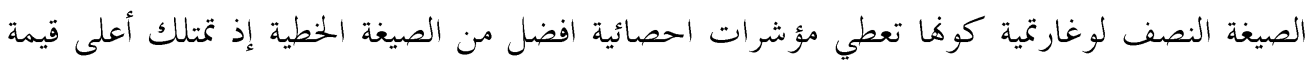

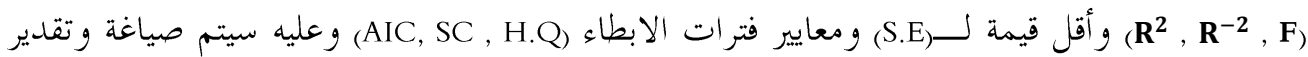
الانموذج القياسي للبحث وفق الصيغة النصف لوغارتمية من اليمين وعلى النحو الاتي:-

inf $=f(\log M, \log x p, \log E x, \log O p, \operatorname{Logoil}, D D) \quad \ldots$

(19)

إذ تم تحويله الى نموذج قياسي نصف لوغاريتمي ليأخذ الشكل الاتي:Inf $=a+b_{1} \log M+b_{2} \log X P+b_{3} \log E x+b_{4} \log O p+b_{5} \log O i l+b_{6} D D+e i$ (20) 


\section{نتائج إختبارات جذر الوحدة لسكون السلاسل الزمنية للمتغيرات محل البحث.}

\section{إختبار ديكي فوللر الموسع (ADF).}

يبين الجحدول (1) نتائج إختبار جذر الوحدة للسلاسل الزمنية للمتغيرات بصيغتها النصف لوغاريتمية، إذ إذ إنسات

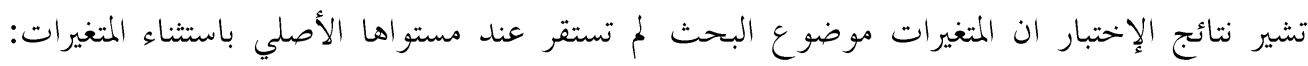
معدل التضخم ومساهمة النفط من الناتج ودرجة الانفتاح الاقتصادي، إذ كانت قيمة (t) المحسوبة أصغر

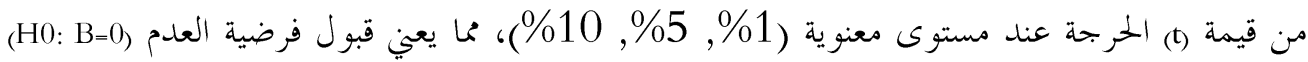

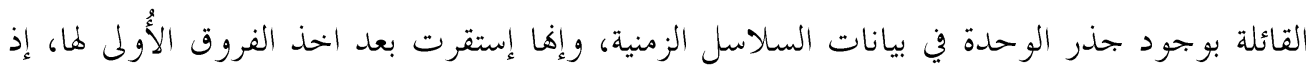

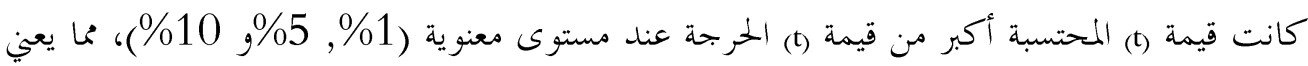

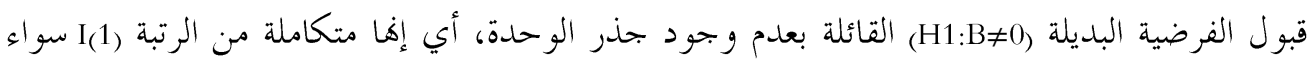

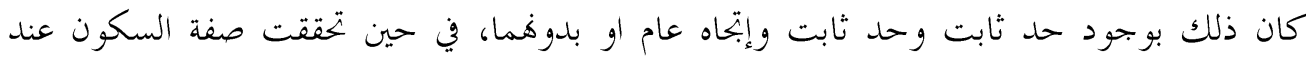
المستوى الأصلي لكل من معدل التضخم ومساهمة النفط من الناتج ودرجة الانفتاح الاقتصادي إذ فمان

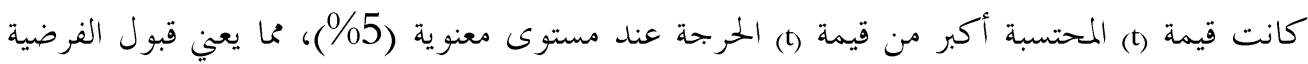

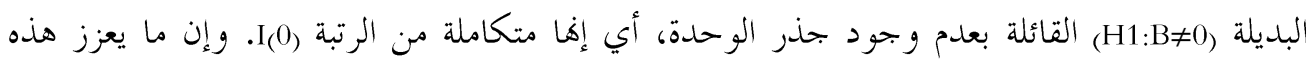

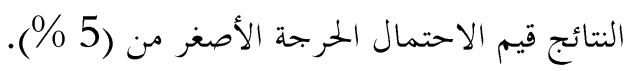

الجدول رقم (1) نتائج إختبار جذر الوحدة حسب إختبار (ADF)

UNIT ROOT TEST TABLE (ADF)

$\begin{array}{lccccccc} & \text { At Level } & & & & & \\ \text { With Constant } & & \text { INF } & \text { LOG(EX) } & \text { LOG(OIL) } & \text { LOG(M2) } & \text { LOG(OP) } & \text { LOG(XP) } \\ & \text { t-Statistic } & -3.2350 & -1.3652 & -3.5074 & -1.6301 & -3.2385 & -1.1903 \\ & \text { Prob. } & \mathbf{0 . 0 2 3 2} & \mathbf{0 . 5 9 2 5} & \mathbf{0 . 0 1 1 5} & \mathbf{0 . 4 6 0 6} & \mathbf{0 . 0 2 3 0} & \mathbf{0 . 6 7 2 4} \\ \text { With Constant \& Trend } & & \star \star & \text { no } & \star \star & \text { no } & \star \star & \text { no } \\ & \text { t-Statistic } & -3.2061 & -1.1037 & -3.7134 & -0.1359 & -3.2019 & -0.4834 \\ & \text { Prob. } & \mathbf{0 . 0 9 3 9} & \mathbf{0 . 9 1 8 9} & \mathbf{0 . 0 2 9 8} & \mathbf{0 . 9 9 3 0} & \mathbf{0 . 0 9 4 7} & \mathbf{0 . 9 8 1 5} \\ \text { Without Constant \& Trend } & & \star & \text { no } & \star \star & \text { no } & \star & \text { no } \\ & \text { t-Statistic } & -3.1201 & 0.0448 & -0.6591 & 4.3131 & -1.1559 & 3.3670 \\ & \text { Prob. } & \mathbf{0 . 0 0 2 4} & \mathbf{0 . 6 9 2 8} & \mathbf{0 . 4 2 7 0} & \mathbf{1 . 0 0 0 0} & \mathbf{0 . 2 2 3 0} & \mathbf{0 . 9 9 9 7} \\ & & \star \star \star & \text { no } & \text { no } & \text { no } & \text { no } & \text { no }\end{array}$

\begin{tabular}{|c|c|c|c|c|c|c|c|}
\hline \multicolumn{8}{|c|}{ At First Difference } \\
\hline & & $\mathrm{d}(\mathrm{INF})$ & $(\operatorname{LOG}(E X))$ & $\mathrm{d}(\mathrm{LOG}(\mathrm{OIL}))$ & $\mathrm{d}(\mathrm{LOG}(\mathrm{M} 2))$ & $d(L O G(O P))$ & $d(\operatorname{LOG}(X P))$ \\
\hline With Constant & $\begin{array}{c}\text { t-Statistic } \\
\text { Prob. }\end{array}$ & $\begin{array}{r}-6.7490 \\
0.0000 \\
\star \star \star \star\end{array}$ & $\begin{array}{c}-3.7352 \\
0.0061 \\
\star \star \star\end{array}$ & $\begin{array}{c}-5.3304 \\
0.0000\end{array}$ & $\begin{array}{c}-6.7075 \\
0.0000\end{array}$ & $\begin{array}{c}-6.3183 \\
0.0000 \\
\star \star \star\end{array}$ & $\begin{array}{c}-10.9130 \\
0.0000 \\
\star \star \star\end{array}$ \\
\hline With Constant \& Trend & $\begin{array}{c}\text { t-Statistic } \\
\text { Prob. }\end{array}$ & $\begin{array}{c}-6.6860 \\
0.0000 \\
\star \star \star\end{array}$ & $\underset{\star \star \star}{-3.8457}$ & $\underset{\star \star \star \star}{-5.2929}$ & $\begin{array}{c}-7.0386 \\
0.0000 \\
\star \star \star\end{array}$ & $\underset{\star \star \star}{-6.2546}$ & $\begin{array}{r}-10.9926 \\
0.0000 \\
\star \star \star\end{array}$ \\
\hline Without Constant \& Trend & $\begin{array}{c}\text { t-Statistic } \\
\text { Prob. }\end{array}$ & 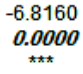 & $\begin{array}{c}-3.4597 \\
0.0009\end{array}$ & $\begin{array}{c}-5.3809 \\
0.0000 \\
\star \star \star\end{array}$ & $\begin{array}{c}-1.8857 \\
0.0572 \\
\star\end{array}$ & $\begin{array}{c}-6.3863 \\
0.0000 \\
\star \star \star\end{array}$ & $\begin{array}{c}-1.4307 \\
0.1405 \\
\text { no }\end{array}$ \\
\hline
\end{tabular}

Notes: $\left(^{\star}\right)$ Significant at the $\left.10 \% ;{ }^{\star \star}\right)$ Significant at the $\left.5 \% ;{ }^{\star \star \star}\right)$ Significant at the $1 \%$. and $($ no $)$ Not Significant *MacKinnon (1996) one-sided p-values. 


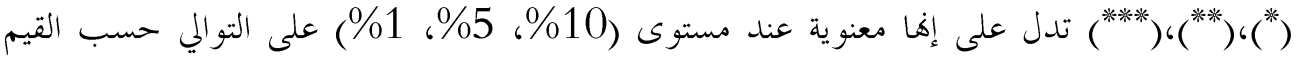

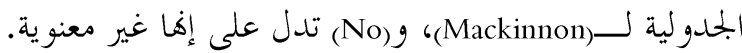

\section{إختبار فيلبس بيرون (PP).}

يلاحظ من خلال الجحدول (2) ان النتائج وفق إختبار فيلبس (PP) لم تختلف كثيراً عما كانت عليه في

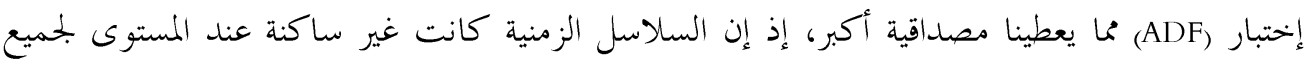

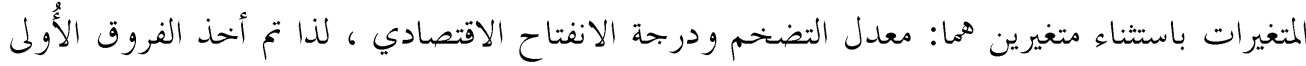

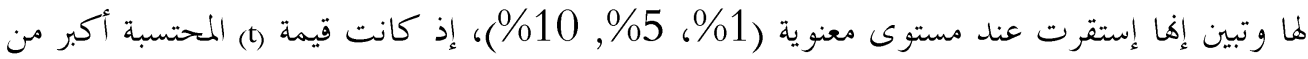

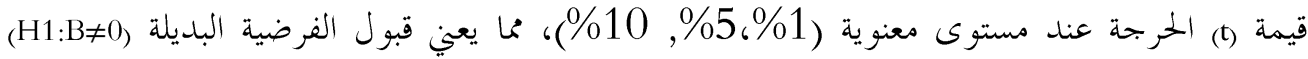

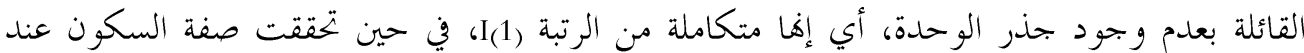

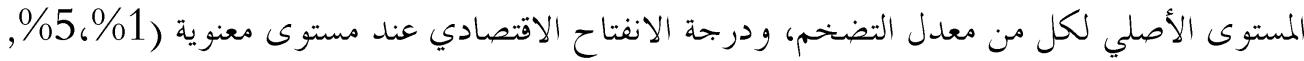

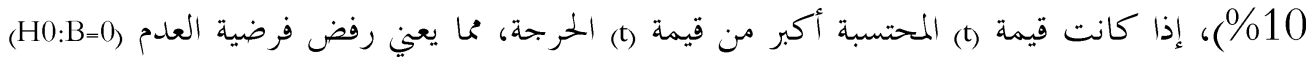

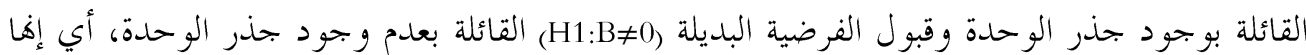

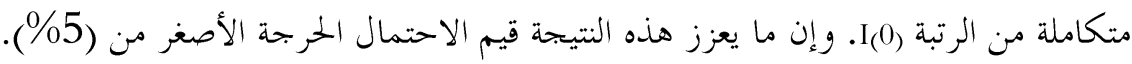

الجدول رقم (2) نتائج إختبار جذر الوحدة حسب إختبار (PP)

\begin{tabular}{|c|c|c|c|c|c|c|c|}
\hline & \multicolumn{7}{|c|}{ UNIT ROOT TEST TABLE (PP) } \\
\hline & 12 & INF & LOG(EX) & LOG(O\|l & LOG(M2) & LOG(OP) & LOG(XP) \\
\hline With Constant & $\begin{array}{c}\text { t-Statistic } \\
\text { Prob. }\end{array}$ & $\begin{array}{c}-3.3884 \\
0.0156 \\
\star \star\end{array}$ & $\begin{array}{c}-1.2222 \\
0.6587 \\
\text { no }\end{array}$ & $\begin{array}{c}-2.6786 \\
0.0842 \\
\star\end{array}$ & $\begin{array}{c}-1.5369 \\
0.5076 \\
\text { no }\end{array}$ & $\begin{array}{c}-3.4053 \\
0.0149 \\
\star \star\end{array}$ & $\begin{array}{c}-1.0835 \\
0.7162 \\
\text { no }\end{array}$ \\
\hline With Constant \& Trend & $\begin{array}{c}\text { t-Statistic } \\
\text { Prob. }\end{array}$ & $\begin{array}{c}-3.3609 \\
0.0674 \\
\star\end{array}$ & $\begin{array}{c}-0.9661 \\
0.9402 \\
\text { no }\end{array}$ & $\begin{array}{c}-2.6947 \\
0.2428 \\
\text { no }\end{array}$ & $\begin{array}{c}-0.3403 \\
0.9874 \\
\text { no }\end{array}$ & $\begin{array}{c}-3.3739 \\
0.0655 \\
\star\end{array}$ & $\begin{array}{c}-1.1498 \\
0.9106 \\
\text { no }\end{array}$ \\
\hline Without Constant \& Trend & $\begin{array}{c}\text { t-Statistic } \\
\text { Prob. }\end{array}$ & $\begin{array}{c}-3.2636 \\
0.0015 \\
\star \star \star\end{array}$ & $\begin{array}{c}0.2277 \\
0.7486 \\
\text { no }\end{array}$ & $\begin{array}{c}-0.3679 \\
0.5475 \\
\text { no }\end{array}$ & $\begin{array}{c}3.0172 \\
0.9992 \\
\text { no }\end{array}$ & $\begin{array}{c}-1.1924 \\
0.2105 \\
\text { no }\end{array}$ & $\begin{array}{c}3.0197 \\
0.9992 \\
\text { no }\end{array}$ \\
\hline & t First D & $\frac{\text { nce }}{d(I N F)}$ & $d(L O G(E X))$ & $d(\mathrm{LOG}(\mathrm{OIL}))$ & $d(L O G(M 2))$ & $d(L O G(O P))$ & $d(L O G(X P))$ \\
\hline With Constant & $\begin{array}{c}\text { t-Statistic } \\
\text { Prob. }\end{array}$ & $\begin{array}{c}-7.3288 \\
0.0000 \\
\star \star \star\end{array}$ & $\begin{array}{c}-3.6466 \\
0.0078 \\
\star \star \star\end{array}$ & $\begin{array}{c}-5.3277 \\
0.0000 \\
\star \star \star\end{array}$ & $\begin{array}{c}-6.8158 \\
0.0000 \\
\star \star \star\end{array}$ & $\underset{* \star}{-6.9845}$ & $\begin{array}{c}-10.8957 \\
0.0000\end{array}$ \\
\hline With Constant \& Trend & $\begin{array}{c}\text { t-Statistic } \\
\text { Prob. }\end{array}$ & $\begin{array}{c}-7.2441 \\
0.0000 \\
\star \star \star\end{array}$ & $\begin{array}{c}-3.7636 \\
0.0264 \\
\star \star\end{array}$ & $\begin{array}{c}-5.3967 \\
0.0002 \\
\star \star \star\end{array}$ & $\begin{array}{c}-7.0553 \\
0.0000\end{array}$ & $\begin{array}{c}-6.9213 \\
0.0000 \\
\star \star \star\end{array}$ & $\begin{array}{c}-11.0426 \\
0.0000 \\
\star \star \star\end{array}$ \\
\hline Without Constant \& Trend & $\begin{array}{c}\text { t-Statistic } \\
\text { Prob. }\end{array}$ & $\begin{array}{c}-7.4165 \\
0.0000 \\
\star \star \star \star\end{array}$ & $\begin{array}{c}-3.3298 \\
0.0013 \\
\star \star \star \star\end{array}$ & $\begin{array}{c}-5.4219 \\
0.0000 \\
\star \star \star \star\end{array}$ & $\begin{array}{c}-5.1520 \\
0.0000 \\
\star \star \star \star\end{array}$ & $\begin{array}{r}-7.0503 \\
0.0000 \\
\star \star \star \star\end{array}$ & $\begin{array}{c}-8.7966 \\
0.0000 \\
\star \star \star\end{array}$ \\
\hline
\end{tabular}




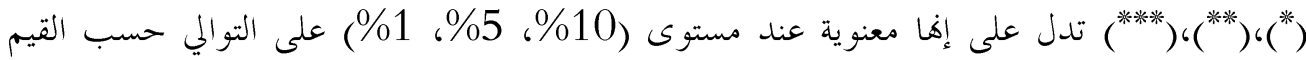
الجحدولية لــ(Mackinnon)، ومo) تدل على إذا غير معنوية.

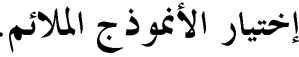

بعد إجراء إختبارات السكون للسلاسل الزمنية لمتغيرات البحث أصبح من المناسب إستخدام منهجية التكامل المشترك وفق أنموذج (ARDL) وتقدير العلاقة التوازنية في الأجلين القصير والطويل، لأن السلاسل الزمنية للمتغيرات محل الدراسة تتصف بالسكون عند درجات مختلفة من المستوى والفرق

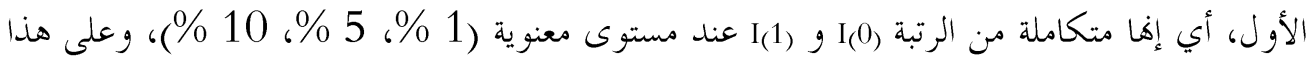
الأساس نستتتج أن أنموذج الإنحدار الذاتي للفجوات الزمنية الموزعة المتباطئة (ARDL) هو الأنسب لقياس وتحليل العلاقة بين المتغيرات موضوع البحث.

\section{صياغة الأنموذج}

بعد إختيار الصيغة النصف لوغاريتمية من اليمين للأكموذج يمكن قياس العلاقة في الأجلين القصير و الطويل وفقاً لأنموذج (ARDL) كما في الصيغة الآتية:A $\quad \Delta \mathbf{I n f}=\mathbf{C}+\mathbf{B}_{\mathbf{1}} \mathbf{I n f}_{\mathbf{t}-\mathbf{1}}+\mathbf{B}_{\mathbf{2}} \log \mathbf{E x}_{\mathbf{t}-\mathbf{1}}+\mathbf{B}_{\mathbf{3}} \log \mathbf{O i l}_{\mathbf{t}-\mathbf{1}}+\mathrm{B}_{4} \log \mathrm{M}_{\mathrm{t}-1}+\mathrm{B}_{5} \log 0 \mathrm{p}_{\mathrm{t}-1}+$ $\mathrm{B}_{6} \log \mathrm{Xp}_{\mathrm{t}-1}+\mathrm{B}_{7} \mathrm{DD}+\sum_{\mathrm{i}=1}^{\mathrm{p}} \lambda_{1} \Delta \operatorname{Inf}_{\mathrm{t}-\mathrm{i}} \sum_{\mathrm{i}=1}^{\mathrm{q}_{1}} \lambda_{2} \Delta \log \operatorname{Ex}_{1 \mathrm{t}-\mathrm{i}}+\sum_{\mathrm{i}=1}^{\mathrm{q}_{2}} \lambda_{3} \Delta \log \operatorname{Oil}_{\mathrm{t}-\mathrm{i}}$ $+\sum_{\mathrm{i}=1}^{\mathrm{q}_{3}} \lambda_{4} \Delta \log \mathbf{M}_{\mathrm{t}-\mathrm{i}}+\sum_{\mathrm{i}=1}^{\mathrm{q}_{4}} \lambda_{5} \Delta \log \mathbf{O} \mathbf{p}_{\mathrm{t}-\mathrm{i}}+\sum_{\mathrm{i}=1}^{\mathrm{q}_{5}} \lambda_{6} \Delta \log \mathbf{X p}_{\mathrm{t}-\mathrm{i}}$

$+\varepsilon t$..... (21)

إذ إن: - n

(inf

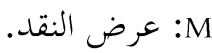
(الإنفاق الاستهلاكي. xp Ex Op 每: مساهمة القطاع النفطي في الناتج المحلي الإجمالي. DD المتغير الوهمي وتم تضمينه لاستبعاد التقلبات التي حصلت خلال مدة البحث.ه: الفروق الأولى لقيم المتغير. C p: علدد ملدد الإبطاء المثلى. 
Bi גi (Et

\section{(ARDL) التقدير الاولي لنموذج}

يبين الجحدول (3) نتائج التقدير الاولي لنموذج مصادر التضخم باستخدام منهجية (ARDL) الذي يوضح العلاقة بين المتغير التابع والمتغيرات المستقلة قيد البحث والذي يستدل منه معنوية النموذج المستخدم في تقدير معلمات الأجل القصير والطويل بحسب المؤشرات الاحصائية F, R-2, R²) كما ان رتبة النموذج الذي تم اختياره وفت منهجية (ARDL) هو (1,2,2,2,2,2,2) بحسب معيار (AIC) والتي تمثل أقل قيمة هذا المعيار والشكل البياني رقم (1) يوضح الرتبة المثلى لانموذج (ARDL) الذي تم اختياره.

Method: ARDL

الجمدول رقم (3) نتائج التقدير الاولى لنموذج مصادر التضخم (ARDL)

Date: $06 / 03 / 20$ Time: $14: 40$

Sample (adjusted): 19722018

Included observations: 47 after adjustments

Maximum dependent lags: 1 (Automatic selection)

Model selection method: Akaike info criterion (AIC)

Dynamic regressors (2 lags, automatic): LOG(EX) LOG(OIL) LOG(M2) LOG(OP) LOG(XP) DD

Fixed regressors: C

Number of models evalulated: 729

Selected Model: ARDL(1, 2, 2, 2, 2, 2, 2)

\begin{tabular}{crrrr}
\hline \hline Variable & Coefficient & Std. Error & t-Statistic & Prob. $^{\star}$ \\
\hline \hline INF(-1) & 0.148207 & 0.088155 & 1.681211 & 0.1043 \\
LOG(EX) & 26.59658 & 14.03371 & 1.895192 & 0.0688 \\
LOG(EX(-1)) & -13.29871 & 21.09658 & -0.630373 & 0.5338 \\
LOG(EX(-2)) & -40.26251 & 16.13063 & -2.496029 & 0.0190 \\
LOG(OIL) & -19.71418 & 6.671024 & -2.955196 & 0.0064 \\
LOG(OIL(-1)) & 17.50279 & 8.339391 & 2.098809 & 0.0453 \\
LOG(OIL(-2)) & -35.54429 & 7.723550 & -4.602067 & 0.0001 \\
LOG(M2) & 20.97803 & 11.57110 & 1.812968 & 0.0810 \\
LOG(M2(-1)) & -20.07579 & 13.12703 & -1.529347 & 0.1378 \\
LOG(M2(-2)) & -34.51985 & 10.58426 & -3.261433 & 0.0030 \\
LOG(OP) & 23.79898 & 2.929726 & 8.123278 & 0.0000 \\
LOG(OP(-1)) & -20.52023 & 3.098171 & -6.623338 & 0.0000 \\
LOG(OP(-2)) & -5.212201 & 3.049748 & -1.709059 & 0.0989 \\
LOG(XP) & 6.032177 & 8.791404 & 0.686145 & 0.4985 \\
LOG(XP(-1)) & 33.85880 & 8.203301 & 4.127460 & 0.0003 \\
LOG(XP(-2)) & 18.27310 & 8.312608 & 2.198239 & 0.0367 \\
DD & 93.35493 & 24.20020 & 3.857609 & 0.0006 \\
DD(-1) & 232.6455 & 32.62547 & 7.130794 & 0.0000 \\
DD(-2) & 147.0629 & 29.83165 & 4.929760 & 0.0000 \\
C & -91.09389 & 53.93827 & -1.688855 & 0.1028 \\
\hline R-squared & 0.975012 & Mean dependent var & 27.44137 \\
Adjusted R-squared & 0.957427 & S.D. dependent var & 93.65837 \\
S.E. of regression & 19.32465 & Akaike info criterion & 9.057393 \\
Sum squared resid & 10082.94 & Schwarz criterion & 9.844690 \\
Log likelihood & -192.8487 & Hannan-Quinn criter. & 9.353658 \\
F-statistic & 55.44775 & Durbin-Watson stat & 1.362063 \\
Prob(F-statistic) & 0.000000 & & & \\
\hline
\end{tabular}


الشكل رقم (1) اختيار الرتبة المثلى لانموذج (ARDL) وفقاً لمعيار (AIC)

Akaike Information Criteria (top 20 models)

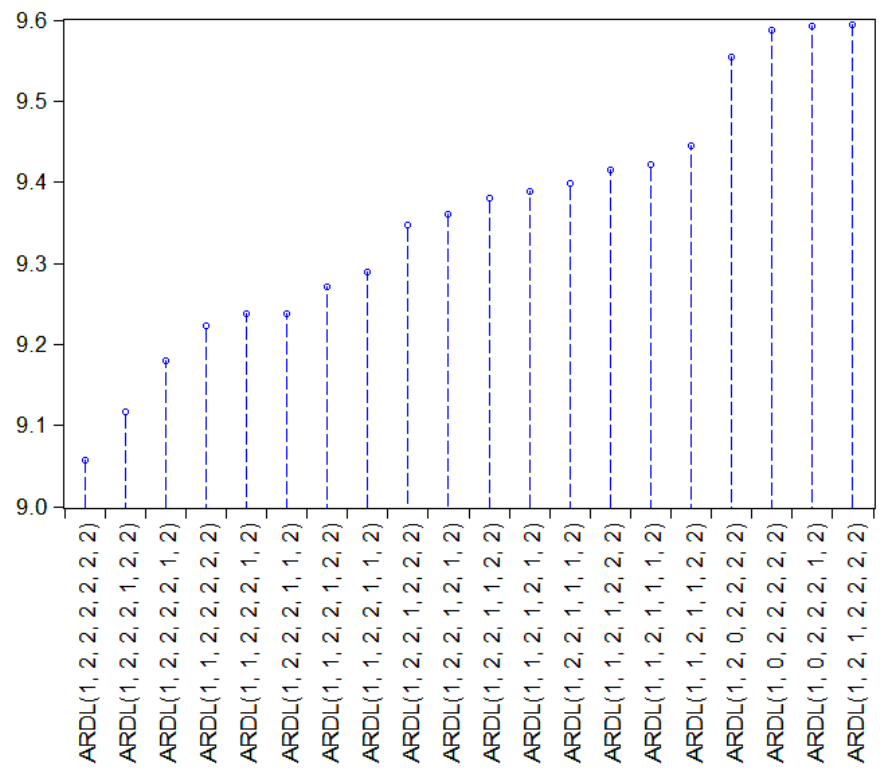

نتائج اختبار التكامل المشترك وفق اختبار الحدود (Bounds Test):

لإختبار ملىى وجود علاقة توازنية طويلة الأجل (وجود تكامل مشترك) بين المتغير التابع والمتغيرات التفسيرية، تم حساب إحصاءة (F) من خلال إختبار الحلدود كما موضح في الجلدول رقم (4) الآتي:

الجدول رقم (4) نتائج إختبار التكامل المشترك للأنموذج المقدر (ARDL) وفق اختبار الحدود

ARDL Bounds Test

Date: 06/03/20 Time: $14: 46$

Sample: 19722018

Included observations: 47

Null Hypothesis: No long-run relationships exist

\begin{tabular}{lll}
\hline \hline Test Statistic & Value & $\mathrm{k}$ \\
\hline \hline F-statistic & 5.254175 & 6 \\
\hline \hline
\end{tabular}

Critical Value Bounds

\begin{tabular}{lcc}
\hline \hline Significance & 10 Bound & I1 Bound \\
\hline \hline $10 \%$ & 2.12 & 3.23 \\
$5 \%$ & 2.45 & 3.61 \\
$2.5 \%$ & 2.75 & 3.99 \\
$1 \%$ & 3.15 & 4.43 \\
\hline \hline
\end{tabular}


يستدل من الجحدول (4) ان قيمة إحصاءة (F) المحتسبة بلغت (5.254) وهي أكبر من القيمة الحرجة للحد

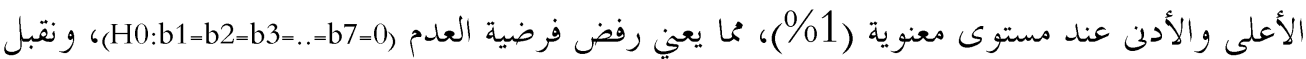

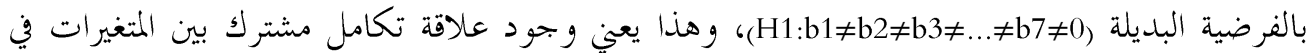
الأنموذج المستخدم للدراسة، أي وجود علاقة توازنية طويلة الأجل تتجه من جميع المتغيرات التفسيرية نغو المتغير التابع، مما يؤكد صحة فرضية البحث محا يستلزم تقدير معلمات الأجلين القصير والطويل و وملمة تصحيح الخطأ.

\section{نتائج تقدير معلمات الأجلين القصير والطويل ومعلمة تصحيح الخطأ}

يستدل من نتائج التقدير في الجمدول (5) و جود علاقة تكامل مشترك بين المتغير التابع (معدل التضخم) والمتغيرات المستقلة وهذا ما يؤكده معامل تصحيح الخطأ (1-1) لهذا النموذج البالغ (-1.35) وان القيمة الاحتمالية (Prob) المصاحبة له هي (0.000) الامر الذي يعني تحقق الشرطين الاساسيين في هذا المعامل و هما قيمته السالبة ومعنويته الاحصائية. مما يعين ان (1.35) من اخطاء الأجل القصير يتم تصحيحها تلقائياً خلال وحدة الزمن (السنة) لبلوغ التوازن في الأجل الطويل اي ان التضخم يتطلب حوالي أقل من سنة (0.7 = 1.35) أي ما يقارب ثمانية اشهر وهي استجابة سريعة لبلوغ قيمته التوازنية في الأجل الطويل بعبارة أخرى ان المدد السابقة تنحرف عن توازن الأجل الطويل وتصحح في المدة الحالية (باعتبارها سرعة تعديل) بنسبة (135 \% \% محا يشير الى ان التكيف كان سريعاً نسبياً. اما نتائج الأجل الطويل فتظهر وجود نتائج عكسية معنوية بين مساهمة القطاع النفطي في الناتج المحلي الاجمالي وعرض النقد الواسع (M) ووعدل التضخم عند مستوى معنوية أقل من (1\%) و استجابة طويلة الأجل عكسية غير معنوية بين سعر الصرف في السوق الموازي ودرجة الانفتاح الاقتصادي ومعدل التضخم فضلاً عن وجود استجابة طويلة الأجل طردية ومعنوية بين الانفاق الاستهالاكي (XP) و معدل التضخم عند مستوى معنوية أقل من (1 \%). 
الجدول رقم (5) نتائج تقدير معلمات الأجلين القصير والطويل ومعلمة تصحيح الخطأ (ARDL) لأنموذج (ARM) لمعدل التضخم ARDL Cointegrating And Long Run Form Dependent Variable: INF

Selected Model: ARDL(1, 2, 2, 2, 2, 2, 2)

Date: $06 / 03 / 20$ Time: $14: 46$

Sample: 19702018

Included observations: 47

\begin{tabular}{crrrr}
\hline \hline \multicolumn{5}{c}{ Cointegrating Form } \\
\hline \hline Variable & \multicolumn{1}{c}{ Coefficient } & Std. Error & t-Statistic & Prob. \\
\hline \hline DLOG(EX) & -0.916806 & 14.033712 & -0.065329 & 0.9484 \\
DLOG(EX(-1)) & 0.570861 & 16.130629 & 0.035390 & 0.9720 \\
DLOG(OIL) & -0.414925 & 6.671024 & -0.062198 & 0.9509 \\
DLOG(OIL(-1)) & 35.544294 & 7.723550 & 4.602067 & 0.0001 \\
DLOG(M2) & 20.978028 & 11.571099 & 1.812968 & 0.0810 \\
DLOG(M2(-1)) & 34.519846 & 10.584256 & 3.261433 & 0.0030 \\
DLOG(OP) & 23.798975 & 2.929726 & 8.123278 & 0.0000 \\
DLOG(OP(-1)) & 5.212201 & 3.049748 & 1.709059 & 0.0989 \\
DLOG(XP) & 6.032177 & 8.791404 & 0.686145 & 0.4985 \\
DLOG(XP(-1)) & -18.273102 & 8.312608 & -2.198239 & 0.0367 \\
D(DD) & 93.354926 & 24.200204 & 3.857609 & 0.0006 \\
D(DD(-1)) & $-147.0629 \ldots$ & 29.831654 & -4.929760 & 0.0000 \\
CointEq(-1) & -1.357205 & 0.088155 & -15.395634 & 0.0000 \\
\hline \hline
\end{tabular}

Cointeq $=$ INF $-\left(-1.6750^{*}\right.$ LOG(EX) $-26.4975^{\star}$ LOG(OIL) $-24.7697^{*}$ LOG(M2)

$-1.4246 *$ LOG $(\mathrm{OP})+42.8558 * \mathrm{LOG}(\mathrm{XP})+348.5570 * \mathrm{DD}-67.1187$ )

\begin{tabular}{crrrr}
\hline \multicolumn{5}{c}{ Long Run Coefficients } \\
\hline \hline Variable & Coefficient & Std. Error & t-Statistic & Prob. \\
\hline \hline LOG(EX) & -1.674982 & 3.912910 & -0.428066 & 0.6720 \\
LOG(OIL) & -26.497453 & 5.843368 & -4.534620 & 0.0001 \\
LOG(M2) & -24.769736 & 8.523332 & -2.906109 & 0.0072 \\
LOG(OP) & -1.424589 & 1.918389 & -0.742597 & 0.4641 \\
LOG(XP) & 42.855781 & 10.840109 & 3.953446 & 0.0005 \\
DD & 348.557038 & 58.047165 & 6.004721 & 0.0000 \\
C & -67.118750 & 41.945420 & -1.600145 & 0.1212 \\
\hline \hline
\end{tabular}

تقييم جودة الأنموذج المقدر اقتصادياً وقياسياً

اولاً: تقييم الأنموذج القياسي المقدر إقتصادياً.

تقييم وتحليل مقدرات معلمات الأنموذج في الأجلين القصير والطويل.

بغية تقييم وتحليل نتائج تقدير معلمات الانموذج القياسي اقتصادياً يتطلب ذلك حساب المرونات الجزئية

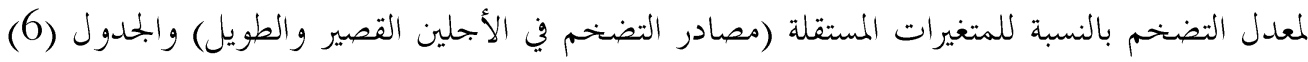

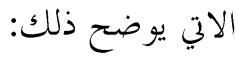


الجحدول رقم (6) المرونات الجزئية للتضخم في الأجلين الطويل والقصير لانموذج (ARDL)

\begin{tabular}{|c|c|c|c|}
\hline المرونة في الأجل الطويل & المرونة في الأجل القصير & التو صيــــــف & 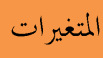 \\
\hline-0.00016 & -8.950 & سعر الصرف في السوق الموازي & EX \\
\hline-0.0025 & -4.048 & مساهمة النفط في الناتج المحلي الاجمالي & Oil \\
\hline-0.0024 & 0.002 & عرض النقد & M \\
\hline-0.00031 & 0.0023 & درجة الانفتاح الاقتصادي & $\mathrm{Op}$ \\
\hline 0.0041 & 0.00058 & الانفاق الاستهلاكي & $\mathrm{XP}$ \\
\hline
\end{tabular}

تم احتساب المرونة الجزئية وفت صيغة الدالة نصف اللوغارتمية من اليمين بحسب الصيغة الاتية:

$$
\mathbf{E i}=\frac{\widehat{B} i}{i \bar{n} f}
$$

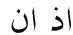

Ei

ق : $\widehat{B} \boldsymbol{i}$

:الوسط الحسابي لمعل التضخم يستنتج من الجمدولين (5) و(6) ما يأني:-

أ. يظهر معامل سعر الصرف في السوق الموازي (EX) إستجابة عكسية وغير معنوية في الأنموذج

المقدر في الأجلين القصير والطويل، إذ بلغت المرونة الجزئية للتضخم بالنسبة لسعر الصرف (8.95-) في الأجل القصير، أي إن زيادة سعر الصرف بنسبة (1\%) يؤدي إلى الخفاض معدل التضخم بنسبة (85.95\%) في الأجل القصير، في حين بلغت المرونة الجزئية في الأنموذج المقدر (00016.0016) في الأجل الطويل، أي ان زيادة سعر الصرف بنسبة (1\%) يؤدي إلى انخفاض معدل التضخم بنسبة (00016\%) في الأجل الطويل. هذه النتيجة كانت متوقعة وتتفق مع منطق النظرية الإقتصادية، لان ارتفاع سعر الصرف يعني ارتفاع قيمة الدينار و انغفاض اسعار الواردات محا يؤدي الى تخفيف الضغط على الاسعار المحلية، كون الاقتصاد العراقي مرتبط بالأسواق الدولية استيراداً وتصديرًا بشكل كبير جداً لان انخفاض المعروض من انتاج الدول الصناعية يسهم في رفع الاسعار العالمية ومن ثم يزيد من معللات التضخم المحلية بسبب اعتماد السوق العراقية في تلبية متطلباها على السوق العالمية. ب. يظهر معامل مساهمة النفط في الناتج المحلي الاجمالي (OIL) استجابة عكسية ومعنوية في الأنموذج المقدر في الأجل الطويل وعكسية وغير معنوية في الأجل القصير، إذ بلغت المرونة 
الجزئية للتضخم بالنسبة للناتج النفطي (4.048-) في الأجل القصير، أي إن زيادة الناتج النفطي بنسبة (1 \%) يؤدي إلى النفاض معدل التضخم بنسبة (4.048 \%) في الأجل

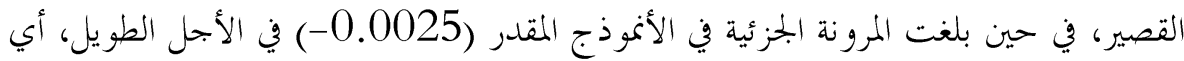

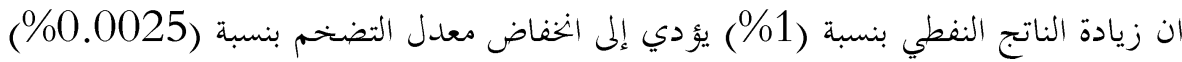
في الأجل الطويل. هذه النتيجة تتفق مع منطق النظرية الإقتصادية ، وذلك بسبب ارتباط اسعار النفط بالسوق العالمية. يظهر معامل عرض النقود (M) إستحابة طردية ومعنوية في الأنموذج المقدر في الأجل القصير وعكسية ومعنوية في الأجل الطويل، إذ بلغت المرونة الجزئية للتضخم بالنسبة لعرض النقد

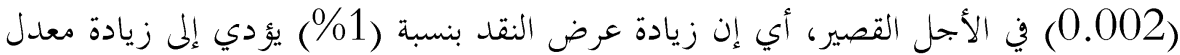
التضخم بنسبة (00202\%) في الأجل القصير، في حين بلغت المرونة الجزئية في الأنموذج

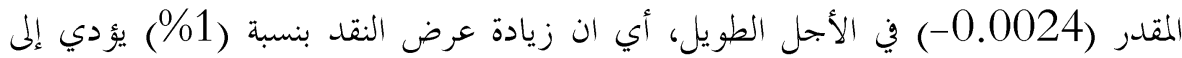
انخفاض معدل التضخم بنسبة (0.0024 \%) في الأجل الطويل. هذه النتيجة كانت غير متوقعة ولا تتفق مع منطق النظرية الإقتصادية، وذلك لحصول البنك المركزي على الاستقلالية كموجب القرار المرقم 56 لسنة 2004. د. يظهر معامل درجة الانفتاح الاقتصادي (OP) إستجابة طردية ومعنوية في الأنموذج المقدر في الأجل القصير وعكسية وغير معنوية في الأجل الطويل، إذ بلغت المرونة الجزئية للتضخم بالنسبة للانفتاح الاقتصادي (0.0023) في الأجل القصير، أي إن زيادة درجة الانفتاح الاقتصادي بنسبة (1 \%) يؤدي إلى زيادة معدل التضخم بنسبة (00230.00\%) في الأجل

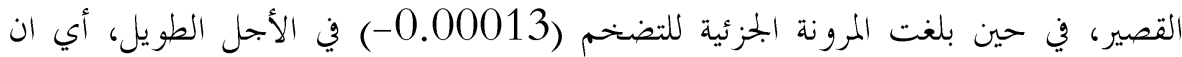

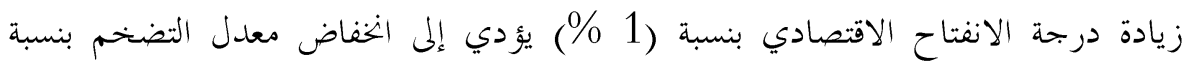
(0.0013 \%) في الأجل الطويل. هذه النتيجة كانت متوقعة وهو يتفق ما توصلت اليه عدد من الدراسات الاقتصادية لان ارتفاع درجة الانفتاح الاقتصادي المحلي للعراق على

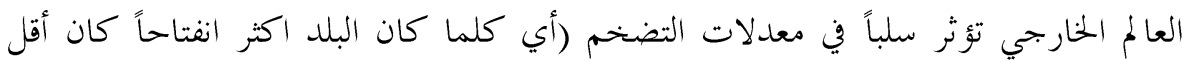
عرضةً للتوسع النقدي غير المتوقع ومن ثم تنخفض معدلات تلات التضخم المحلية). ه. يظهر معامل الانفاق الاستهلاكي (XP) إستجابة طردية وغير معنوية في الأنموذج المقدر في مئه الأجل القصير وطردية ومعنوية في الأجل الطويل، إذ بلغت المرونة الجزئية للتضخم بالنسبة 
للانفاق الاستهلاكي (0.00058) في الأجل القصير، أي إن زيادة الانفاق الاستهلاكي بنسبة (1 \%) يؤدي إلى زيادة معدل التضخم بنسبة (0058\%) في الأجل القصير، في حين بلغت المرونة الجزئية (0.0041) في الأجل الطويل، أي ان زيادة الانفاق الاستهلاكي

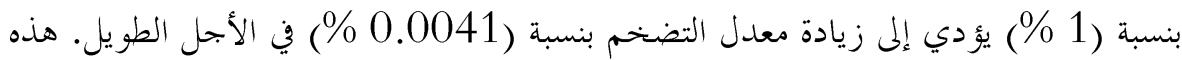
النتيجة كانت متوقعة وتتفق مع منطق النظرية الإقتصادية، اما كوها غير معنوية فان التغيرات في كمية النقود في الأجل القصير لا يظهر اثرها على التضخم كونه يتم حسابه على اساس سنوي. و. يظهر معامل المتغير الوهمي إستحابة طردية ومعنوية في الأنموذج المقدر في الأجلين القصير والطويل، هذه النتيجة كانت متوقعة وتتفق مع منطق النظرية الإقتصادية، كونه يمثل استبعاد

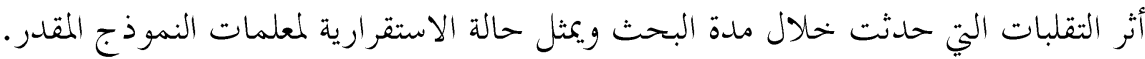

\section{ثانياً: تقييم الأنموذج المقدر قياسياً}

للتأكد من جودة الأنموذج المستخدم في القياس والتحليل وخلوه من المشاكل القياسية، يستلزم إجراء الإختبارات التشخيصية الآتية:أ- إختبار الإرتباط الذاتي Autocorrelation): ويتم ذلك من خلال إختبار مضروب لاكرانج للإرتباط التسلسلي (BGLM):- يلاحظ من الجلدول (7) ان قيمة إحصاءة (F) المحتسبة بلغت (1.101) بقيمة إحتمالية (Pro: 0.399)، وعليه نقبل فرضية العدم القائلة بخلو الأنموذج المقدر من مشكلة الإرتباط $(H 0: \rho=0)$

الجدول رقم (7) نتائج إختبار (BGLM) للأثموذج المقدر

\section{Breusch-Godfrey Serial Correlation LM Test:}

\begin{tabular}{llll}
\hline \hline F-statistic & 1.101506 & Prob. F(7,20) & 0.3996 \\
Obs*R-squared & 13.07789 & Prob. Chi-Square(7) & 0.0702 \\
\hline \hline
\end{tabular}

كما يظهر اختبار معاملات الارتباط الذاتي عدم وجود ارتباط ذاتي في بواقي النموذج وهي ضمن حدود الثقة (5\%) وعدم معنوية معاملات الارتباطات الذاتية والجزئية كما يؤكد ذلك اختبار رسم الارتباط واختبار قيمة Q في الجدول التالي:- 


$$
\text { الجحدول رقم (8) معاملات الارتباط الذاتي والجزئي لبو اقي الانموذج المقدر }
$$

Date: $06 / 03 / 20$ Time: $14: 49$

Sample: 19702018

Included observations: 47

\begin{tabular}{|c|c|c|c|c|c|c|c|}
\hline Autocorrelation & Partial Cor & rrelation & & $\mathrm{AC}$ & PAC & Q-Stat & Prob* \\
\hline $1 \models$ & $1 E$ & $\square$ & 1 & 0.298 & 0.298 & 4.4477 & 0.035 \\
\hline $1 \square \quad 1$ & & 1 & 2 & -0.196 & -0.312 & 6.4102 & 0.041 \\
\hline 101 & 10 & 1 & 3 & -0.094 & 0.092 & 6.8764 & 0.076 \\
\hline 1 ا & 17 & 1 & 4 & 0.122 & 0.081 & 7.6750 & 0.104 \\
\hline 151 & 1 & 1 & 5 & 0.070 & -0.029 & 7.9413 & 0.160 \\
\hline 101 & 10 & 1 & 6 & -0.101 & -0.073 & 8.5100 & 0.203 \\
\hline 1 만 & 10 & 1 & 7 & -0.186 & -0.124 & 10.491 & 0.162 \\
\hline 10 & 10 & 1 & 8 & -0.129 & -0.088 & 11.476 & 0.176 \\
\hline 1 은 & 1영 & 1 & 9 & -0.143 & -0.181 & 12.718 & 0.176 \\
\hline 10 & 1 & 1 & 10 & -0.076 & -0.007 & 13.081 & 0.219 \\
\hline 1 & I & 1 & 11 & -0.002 & -0.021 & 13.081 & 0.288 \\
\hline 10 & 10 & 1 & 12 & -0.076 & -0.119 & 13.461 & 0.336 \\
\hline 1 & $1 \mathrm{~b}$ & 1 & 13 & -0.031 & 0.054 & 13.527 & 0.408 \\
\hline 1 & 10 & 1 & 14 & -0.028 & -0.126 & 13.583 & 0.481 \\
\hline 101 & 10 & 1 & 15 & -0.070 & -0.111 & 13.932 & 0.531 \\
\hline 101 & 10 & 1 & 16 & -0.091 & -0.135 & 14.542 & 0.558 \\
\hline 1 & 1 & 1 & 17 & -0.001 & -0.036 & 14.542 & 0.628 \\
\hline 1 & 1 & 1 & 18 & 0.118 & 0.033 & 15.645 & 0.617 \\
\hline 101 & 1 & 1 & 19 & 0.075 & -0.044 & 16.103 & 0.650 \\
\hline 101 & 10 & 1 & 20 & -0.062 & -0.062 & 16.436 & 0.689 \\
\hline
\end{tabular}

ب-إختبار عدم ثبات التباين المشروط بالإنحدار الذاتي (ARCH):- يلاحظ من الجلدول (9) ان قيمة إحصاءة (F) المحتسبة بلغت (Pro: 0.505) (Pro)، وهذا يعين قبول فرضية العدم (مستوى إحتمال القائلة بثبات تباين حد الخطاً العشوائي في الأنموذج المقدر

$$
\text { الجحدول رقم (9) نتائج إختبار ARCH) للأكموذج المقدر }
$$

Heteroskedasticity Test: ARCH

\begin{tabular}{llll}
\hline \hline F-statistic & 0.451675 & Prob. F(1,44) & 0.5051 \\
Obs*R-squared & 0.467407 & Prob. Chi-Square(1) & 0.4942 \\
\hline \hline
\end{tabular}

Test Equation:

Dependent Variable: RESID 2

Method: Least Squares

Date: 06/03/20 Time: $14: 49$

Sample (adjusted): 19732018

Included observations: 46 after adjustments

\begin{tabular}{lrlll}
\hline \hline \multicolumn{1}{c}{ Variable } & Coefficient & Std. Error & t-Statistic & Prob. \\
\hline \hline \multicolumn{1}{c}{ C } & 234.2856 & 55.37947 & 4.230549 & 0.0001 \\
\multicolumn{1}{c}{ RESID $2(-1)$} & -0.100821 & 0.150015 & -0.672068 & 0.5051 \\
\hline \hline R-squared & 0.010161 & Mean dependent var & 212.4284 \\
Adjusted R-squared & -0.012335 & S.D. dependent var & 302.1527 \\
S.E. of regression & 304.0105 & Akaike info criterion & 14.31451 \\
Sum squared resid & 4066586. & Schwarz criterion & 14.39401 \\
Log likelihood & -327.2336 & Hannan-Quinn criter. & 14.34429 \\
F-statistic & 0.451675 & Durbin-Watson stat & 2.007122 \\
Prob(F-statistic) & 0.505055 & & & \\
\hline
\end{tabular}


جـ- اختبار التوزيع الطبيعي للأخطاء العشوائية (J-B): بلغت قيمة إحصاءة اختبار (JB) (1.238) بقيمة احتمالية (Prob=0.538) مما يعين قبول فرضية العدم القائلة بأن الأخطاء العشوائية تتوزع توزيعاً طبيعياً في الأكموذج المقدر (ARDL) (

\section{نتائج إختبار الإستقرارية الهيكلية لمعاملات أنموذج (ARDL)}

بعد تقدير صيغة تصحيح الخطأ لأنموذج (ARDL)، يستلزم إجراء إختبار الإستقرار الهيكلي لمعاملات الأجلين القصير والطويل لأنموذج التضخم للتأكد من خلو البيانات المستخدمة من وجود أي تغيرات هيكلية فيها، ويتم ذلك من خلال إختبارين هما: إختبار المجموع التراكمي للبو اقي المعاودة (CUSUM)، وإختبار المجموع التراكمي لمربعات البو اقي المعاودة (CUSUM-SQ) كما مبين في الشكل الآتي:

الشكل رقم (2) الإستقر ارية الهيكلية لمعاملات أنموذج التضخم المقدر
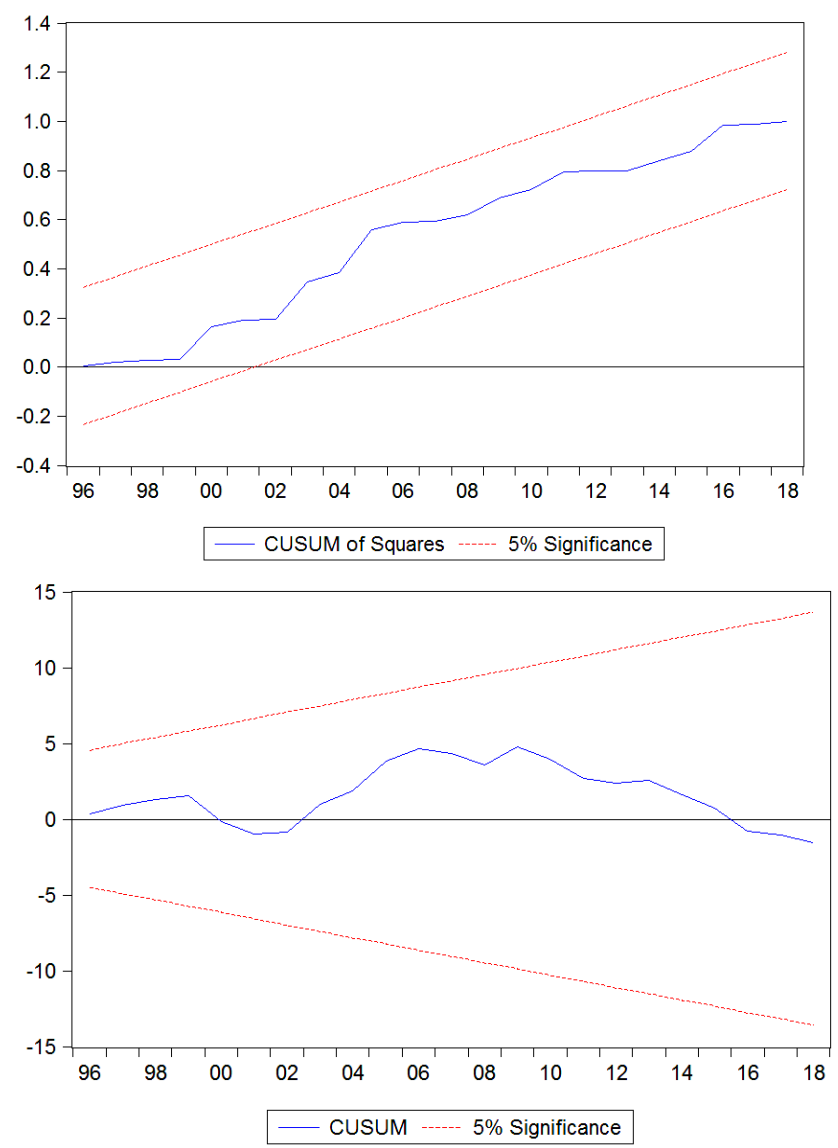
يلاحظ من الشكل أعلاه ان إحصاءة إختبار المجموع التراكمي للبواقي المعاودة CUSUM) ولمربعات البو اقي المعاودة (SUSUM-SQ) وقع داخل الحدود الحرجة (الحد الأعلى و الحد الأدنى) عند مستوى

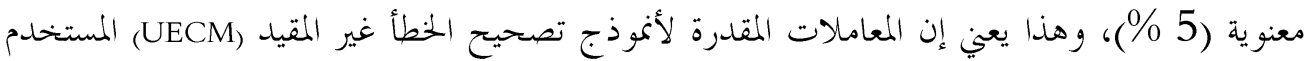

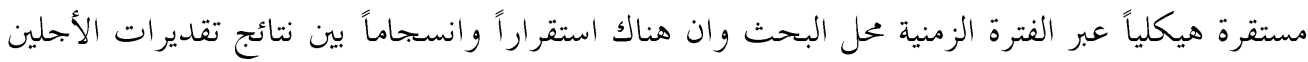
القصير و الطو يل.

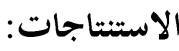

من خحلال ما تقدم يمكن التوصل إلى الاستنتاجات الآتية:

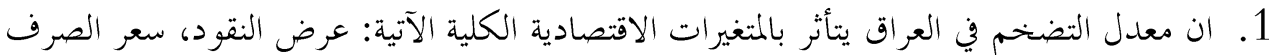
في السوق الموازي ، الإنفاق الاستهلاكي، مساهمة القطاع النفطي في الناتج المحلي الاجمالي ،

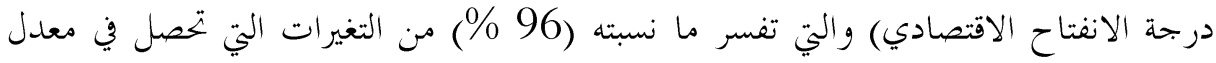
التضخم وكلها ترتبط بعلاقة عكسية مع معدل التضخم باستثناء عرض النقود والانفاق

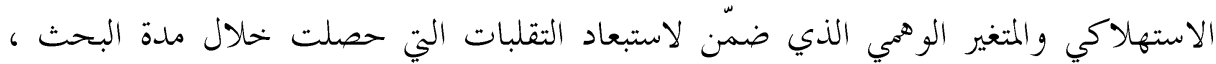
كما توجد علاقة توازنية طويلة الأجل (تكامل مشترك) بين المتغيرات المدروسة وفق منهجية إختبار الحدود (Bound Test). 2. تبين ان قيمة معامل تصحيح الخطأ تساوي (1.35 -) وهي سالبة وذات دلالة احصائية عند مستوى معنوية أقل من (1 \%) وهذا يعني ان (1.35) من اخطاء الاجل القصير يتم تصحيحها

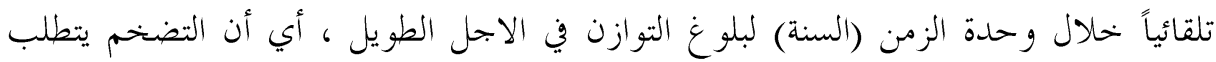

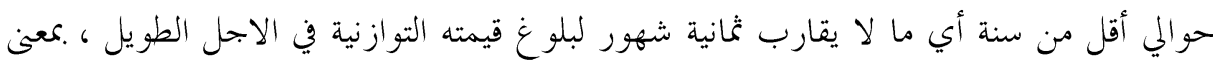
آخر ان انخراف السنوات السابقة عن توازن الاجل الطويل يتم تصحيحها بنسبة (135\%). 3. يظهر الإختبارين المجموع التراكمي للبواقي المعاودة (CUSUM) و المجموع ع التراكمي لمربعات

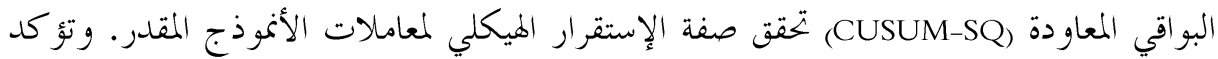
نتائج الدراسة خلو الأنموذج المقدر من مشاكل التحليل القياسي. التوصيات: حاول الباحث ان يساهم بإبداء التوصيات لعلها تفيد في معالجة التضخم في الاقتصاد العراقي ولعل أهم

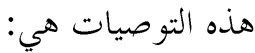


1. أهمية التحكم في كمية النقود المتداولة وترشيد الإصدار النقدي باتجاه تحقيق أكبر قدر من التناسب بين متوسط نمو الناتج المحلي الإجمالي، والنمو في كمية النقود المتداولة بما يضمن تحقيق التوازن بين القطاعين النقدي والحقيقي لبلوغ استقرار الرقم القياسي للاسعار. 2. ضرورة التوسع في الائتمان المصرفي عن طريق وضع نظام عقلاني للرقابة على الائتمان المصرفي

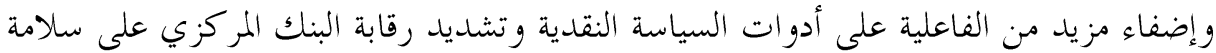
وتوزيع التسهيلات المصرفية للقطاع الخاص وفرض حدود قصوى للتسهيلات الائتمانية للوحدات المقترضة. 3. ربط التوسع في الاستخدام عبر التوسع في القدرات الإنتاجية المحلية من جهة والتوسع في القدرات التكنولوجية والتعليمية والتدريبية من جهة أخرى بما يعزز تكوين المهارات وتعظيم التهاتيه كفاءة الأداء بما يساعد على زيادة إنتاجية العمل، ودمج قطاع النفط مع القطاعات الاقتصادية الأخرى في توجه مخطط طويل المدى يهدف إلى تحضير الاقتصاد العراقي لليوم الذي ينضب فيه النفط مع ضرورة ربط الأجر بالإنتاجية. 4. قيام السلطة النقدية بالعمل على تقليص كمية العملة في التداول وزيادة الودائع الجحارية ومكافحة السوق الموازي للعملة من خلال تقليل الفرق بين السعر الرسمي والسعر الموازي للصرف والعمل على جذب العملة داخل المصارف من خلال تشجيع التعامل بالحسابات الجارية وتوسيع قاعدة التعاملات المصرفية وتنمية العادات الادخارية وتعزيز ثقة الافراد بالعملة الوطنية. 1. ابدجمان ، مايكل ابدجمان ، مايكل الاقتصاد الكلي ، النظرية والسياسات ، ترجمة عمد ابراهيم منصور ، مراجعة عبد الفتاح عبد الرحمن ،

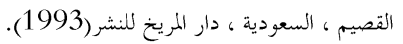
2. اكلي الاقتصاد الكلي ، النظرية والسياسات ، ترجمة عطبة مهدي سليمان ، مراجعة : عبد المنعم السيد علي(1980)،

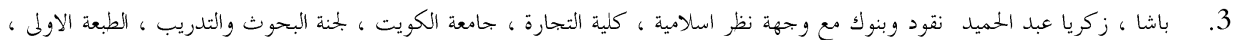
الكويت(1989). 4. الفضيل ، محمد عبد ، مشكلة التضخم في الاقتصاد العربي ، الجذور والمسببات والابعاد والسياسات ، دار المتوكل للنشر - بيروت (1982). 5. شيخي محمد , طرق الاقتصاد والقياس , محاضرات وتطبيقات , ط1, دار الحامد للنشر , عمان (2012). 6. الدليمي , مالك علام عفات , قياس وتحليل محددات الطلب على النقود في الاقتصاد العراقي للمدة (2015- 1985) , رسالة ماجستير (غير منشورة) , كلية الإدارة والاقتصاد , جامعة الفلوجة (2018). العيسى , سلوى عبدالرحمن , , أثر الانفاق اللكومي على النمو الاقتصادي في المملكة العربية السعودية والكويت والإمارات العربية المتحدة، .7

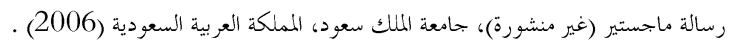
8. الفراج فراج عبد العزيز ، العلاقة بين المؤشر العام لسوق الاسهم المحلية وبعض المتغيرات الاقتصادية الكلية في المملكة العربية السعودية، رسالة ماجستير, كلية العلوم الادارية، جامعة الملك سعود، الرياض (2004). 
سليمان , عمر إبراهيم عناد، مسار الانفاق العام ومدى مساهته في تحقيق النمو الاقتصادي في العراق للمدة (2015-2004)رسالة ماجستير

$$
\text { (غير منشورة) , كلية الإدارة والاقتصاد , جامعة الفلوجة (2017). }
$$

10. العيثاوي ، مصطفى اسماعيل جاسم ، قياس وتحليل الصدمات الخنارجية واثرها على بعض المتغيرات الاقتصادية الكلية في الاقتصاد العراقي للمدة

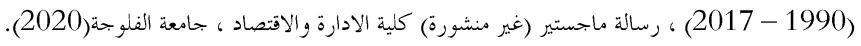

11. الزهراني ، بندر بن سالم ، الاستثمارات الاجنبية المباشرة ودورها في النمو الاقتصادي في المملكة العرية السعودية ، دراسة قياسية للمدة

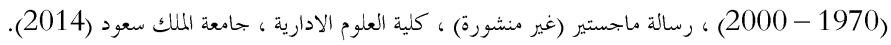

12. الصبيحي ، علي نبع مشكلة التضخم في الاقتصاد اليمني 2000 - 2004 ، بحلة كلية العلوم التطبيقية ، جامعة حضر موت للعلوم

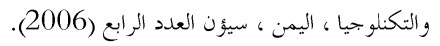

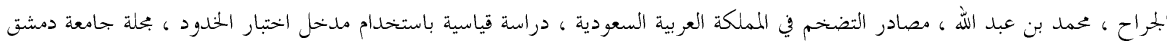

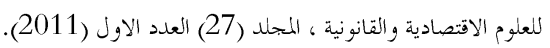

14. رشاد , ندوى خزعل (2011) , استخدام اختبار كرانجر في تحليل السلاسل الزمنية المستقرة, المجلة العراقية للعلوم الاقتصادية , الجامعة

$$
\text { المستنصرية , العدد (9) , بغداد. }
$$

القدير , خالد , العلاقة بين كمية النقود والناتج المحلي الإجمالي في قطر , دراسة تطبيقية باستخدام التكامل المشترك , بحلة الدراسات الاقتصادية

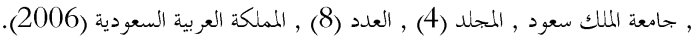

بتال , أحمد حسين , حيدر ال طعمة , عبدالرحمن عبيد , دور التطور الملاي في تحقيق النمو الاقتصادي (العراق حالة دراسية) , مؤتمر جامعة

نوروز الدولي الثاني , جامعة نوروز , دهوك - كردستان العراق (2014).

عبد القادر ، سيد متولي (2007) ، اشتقاق إنموذج تصحيح الخطأ من اختار التكامل المشترك بلموهانسن ، اطار نظري ومثال تطبيقي باستخدام

$$
\text { برنامج (E. 5. منتديات الاحصائيين العرب. }
$$

العبدلي , عابد بن عابد, تقدير محددات كل واردات المملكة العربية السعودية في إطار التكامل المشترك وتصحيح الخطاً , بحلة مركز صالح عبدالله

$$
\text { كامل للاقتصاد الإسلامي , العدد (32). }
$$

الجنابي ، نبيل مهدي (2012) ، السياسة النقدية والمالية مع تطبيق معادلة (2t.louis) على الاقتصاد العراقي للمدة (2003 - 2011) ، مبحلة

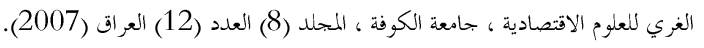

الشوريجي , بمحدي, أثر النمو الاقتصادي على العمالة الاقتصادية في الاقتصاد المصري, بحلة اقتصاديات شمال أفريقيا , العدد (6) , مصر,

حسن , علي عبدالزهرة, تحليل العلاقة التوازنية طويلة الأجل باستعمال اختبارات جذر الوحدة واسلوب دمج النماذج المرتبطة ذاتياً ونماذج

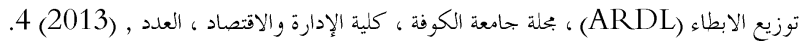

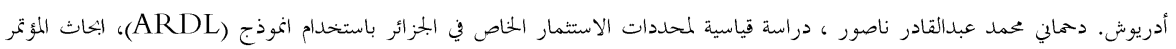

الدولي، جامعة سطيف، الجزائر (2013).

عطية، عبدالقادر محمد عبدالقادر , الاقتصاد القياسي بين النظرية والتطبيق، الدار الجامعية , مصر (2005).

• داغر محمود محمد، الاقتصاد الكلي نظريات وسياسات ، الطبعة الأولى ، دار الكتب والوثائق ، بغداد، 2018. 